\title{
Beneficial biological effects and the underlying mechanisms of molecular hydrogen - comprehensive review of 321 original articles -
}

\author{
Masatoshi Ichihara', Sayaka Sobue ${ }^{1}$, Mikako Ito ${ }^{2}$, Masafumi Ito ${ }^{3}$, Masaaki Hirayama ${ }^{4}$ and Kinji Ohno ${ }^{2 *}$
}

\begin{abstract}
Therapeutic effects of molecular hydrogen for a wide range of disease models and human diseases have been investigated since 2007. A total of 321 original articles have been published from 2007 to June 2015. Most studies have been conducted in Japan, China, and the USA. About three-quarters of the articles show the effects in mice and rats. The number of clinical trials is increasing every year. In most diseases, the effect of hydrogen has been reported with hydrogen water or hydrogen gas, which was followed by confirmation of the effect with hydrogen-rich saline. Hydrogen water is mostly given ad libitum. Hydrogen gas of less than $4 \%$ is given by inhalation. The effects have been reported in essentially all organs covering 31 disease categories that can be subdivided into 166 disease models, human diseases, treatment-associated pathologies, and pathophysiological conditions of plants with a predominance of oxidative stress-mediated diseases and inflammatory diseases. Specific extinctions of hydroxyl radical and peroxynitrite were initially presented, but the radical-scavenging effect of hydrogen cannot be held solely accountable for its drastic effects. We and others have shown that the effects can be mediated by modulating activities and expressions of various molecules such as Lyn, ERK, p38, JNK, ASK1, Akt, GTP-Rac1, iNOS, Nox1, NF-kB p65, IkBa, STAT3, NFATC1, c-Fos, and ghrelin. Master regulator(s) that drive these modifications, however, remain to be elucidated and are currently being extensively investigated.
\end{abstract}

Keywords: Molecular hydrogen, Ischemia-reperfusion injury, Inflammatory diseases

\section{Introduction}

It has been 8 years since Ohsawa and colleagues reported the astonishing therapeutic effects of molecular hydrogen on a rat model of cerebral infarction in Nature Medicine in 2007 [1]. Inhalation of 1-4 \% hydrogen gas markedly reduced the sizes of cerebral infarction in rats. They also demonstrated that hydrogen specifically scavenges hydroxyl radical and peroxynitrite but not hydrogen peroxide or superoxide. Their paper ignited interest in the effect of molecular hydrogen in various diseases and has been cited 533 times as of July 2015. Similarly, the number of original articles demonstrating the effect of molecular hydrogen adds up to more than 300. This review summarizes research articles published in these

\footnotetext{
* Correspondence: ohnok@med.nagoya-u.ac.jp

${ }^{2}$ Division of Neurogenetics, Center for Neurological Diseases and Cancer, Nagoya University Graduate School of Medicine, 65 TsurumaiShowa-ku, Nagoya 466-8550, Japan

Full list of author information is available at the end of the article
}

past 8 years and addresses possible molecular mechanisms underlying the effects of hydrogen.

\section{Molecular hydrogen research before 2007}

Even before the publication by Ohsawa and colleagues in 2007 [1], biological effects of molecular hydrogen had been investigated in a small scale, as shown below. Dole and colleagues first reported the hydrogen effect in Science in 1975 [2]. They placed nude mice carrying squamous cell carcinoma in a chamber with $2.5 \%$ oxygen and $97.5 \%$ hydrogen under 8-atmospheric pressure and observed prominent reduction in the size of the tumors. A similar effect of hyperbaric hydrogen on leukemia was reported in 1978 [3]. Hydreliox, which contained $49 \%$ hydrogen, $50 \%$ helium, and $1 \%$ oxygen, was reported to be effective to prevent decompression sickness and nitrogen narcosis for divers working below 500 meters under sea level [4]. An anti-inflammatory effect of hyperbaric hydrogen on a mouse model of schistosomiasis-associated chronic liver 
inflammation was also reported in 2001 [5]. Hyperbaric hydrogen may be effective for some diseases, but only a limited number of studies have been published. The difference between hyperbaric and normobaric hydrogen has not been directly compared to date.

Following a small number of studies with hyperbaric hydrogen, the effect of electrolytically alkaline water has been reported. Shirahata and colleagues hypothesized that the hydrogen atom, which they called active hydrogen, is generated in electrolysis and proposed that active hydrogen scavenges reactive oxygen species (ROS) [6]. Although it is unlikely that atomic hydrogen is able to exist for a substantial time in our bodies, molecular hydrogen does exist in electrolyzed water and the effects of electrolyzed water have been reported thereafter. $\mathrm{Li}$ and colleagues reported that electrolyzed water scavenged ROS and protected a hamster pancreatic beta cell line from alloxan-induced cell damage [7]. Similarly, reduced hemodialysis solution produced by an electrolysis device (Nihon Trim Co. Ltd.) ameliorated oxidative stress in hemodialysis patients [8]. In 2005, researchers in Tohoku University Graduate School of Medicine and Nihon Trim started cooperative clinical studies and established the Association of Electrolyzed WaterHemodialysis Study Group in 2008. According to personal communications with this group, they now believe that the effects of electrolyzed water are likely due to dissolved hydrogen molecules.

In 2005, Yanagihara and colleagues at Miz Co. Ltd. reported that hydrogen-rich neutral water that was produced with their unique electrolysis device reduced oxidative stress in rats [9]. This was a pioneering work, because they explicitly proved that molecular hydrogen but not alkaline in the electrolyzed alkaline water exerts therapeutic effects.

\section{Molecular hydrogen research in and after year 2007}

As stated in the introduction, the Nature Medicine paper in 2007 [1] spurred interest in hydrogen research. Figure 1 shows 321 original articles up to June 2015 in the MEDLINE database, which demonstrate the effects of molecular hydrogen on disease models, human diseases, treatment-associated pathologies, and pathophysiological conditions of plants. Most studies were conducted in Japan, China, and the USA, with a predominance of China since 2010 (Fig. 1A). About threequarters of the articles show the effects in mice and rats (Fig. 1B), but the number of human studies is increasing every year ( 1 article each in 2008-2009; 2 in 2010; 3 in 2011; 5 in 2012; 9 in 2013; 6 in 2014; and 6 in 2015). In addition, the effects of hydrogen have been reported in plants in 13 articles, which suggest a wide range of effects over various species not restricted to mammals. The effects of molecular hydrogen on plants may

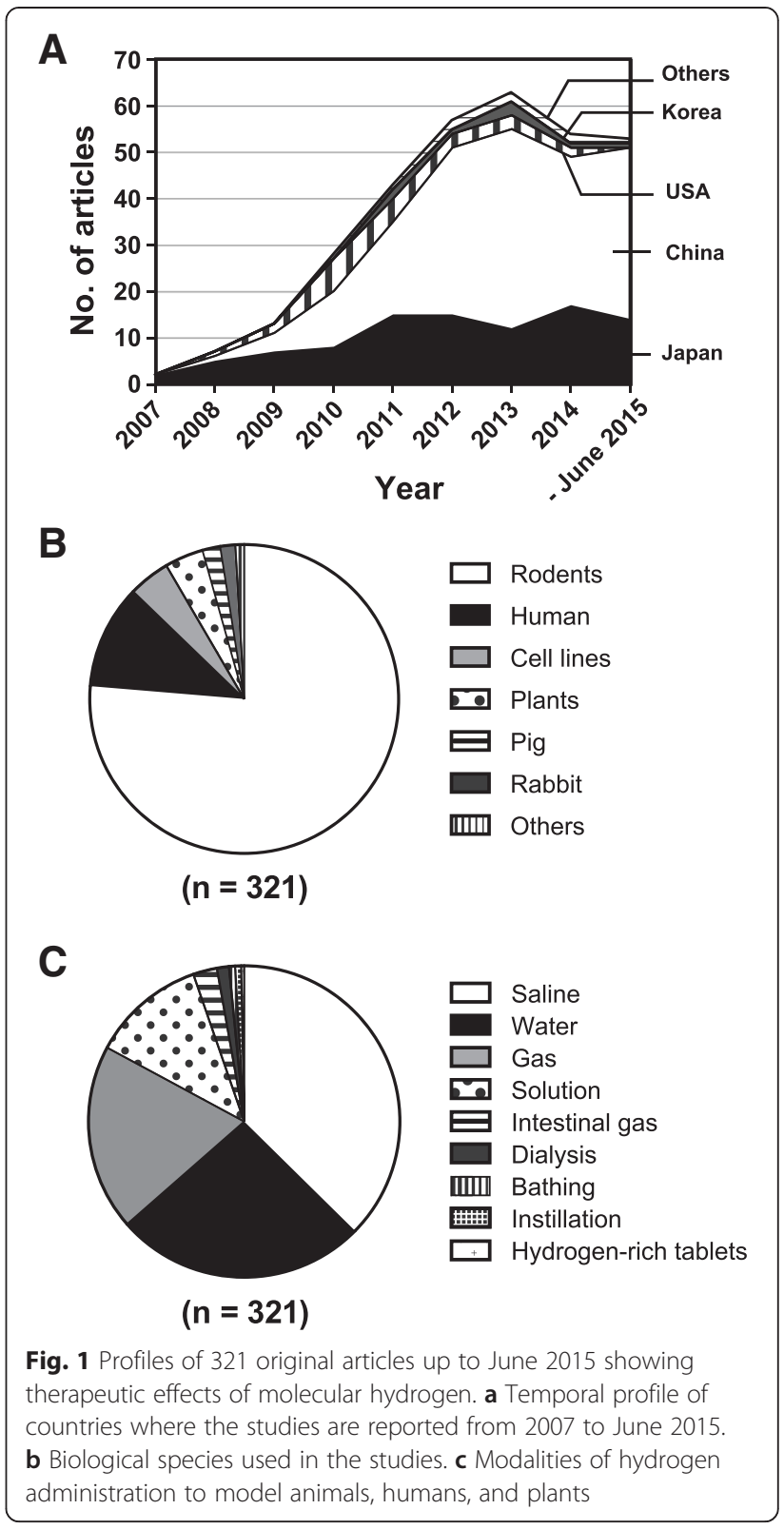

warrant application of hydrogen to increase agricultural production. Modalities of hydrogen administration are shown in Fig. 1C. Hydrogen-rich saline, which is almost exclusively used in China, dominates over the others. Hydrogenized saline is administered either by intraperitoneal injection or drip infusion. Hydrogen water is mostly given ad libitum. Hydrogen gas is usually given by inhaling $1-4 \%$ hydrogen gas, which is below the explosion level $(4 \%)$. There is a single report, in which hydrogen gas was injected intraperitoneally [10].

Among the various routes of hydrogen administration shown in Fig. 1C, the best method still remains uncertain. This is partly because only a few reports have addressed the difference of effects among administration 
methods. We previously showed that drinking hydrogen water, but not continuous hydrogen gas exposure, prevented development of 6-hydorxydopamine-induced Parkinson's disease in rats [11]. In addition, we recently showed that continuous exposure to hydrogen gas and ad libitum per os administration of hydrogen water modulated signaling pathways and gene expressions in different manners in mice [12]. We demonstrated that hydrogen-responsive genes are divided into four groups: genes that respond favorably to hydrogen gas, those that respond exclusively to hydrogen water, those that respond to both hydrogen gas and water, and those that respond only to the simultaneous administration of gas and water (Fig. 2). As hydrogen gas and water increase the hydrogen concentrations in the rodent body to a similar level [12], the difference in the organs exposed to a high concentration of hydrogen, the rise time of hydrogen concentration, and/or the area under the curve of hydrogen concentration may account for the difference in the modulated genes. On the other hand, a collation of hydrogen reports indicate that a similar degree of effects can be observed with different modalities of administration. For example, the marked effect of hydrogen on a mouse model of LPS-induced acute lung injury has been reported by four different groups with three different modalities: hydrogen gas [13, 14], hydrogen water [15], and hydrogen-rich saline [14, 16]. Similarly, the dramatic effect of hydrogen on animal models of acute myocardial infarction has been reported by eight different groups with two different modalities: hydrogen gas [17-20] and hydrogen-rich saline [21-24]. To clarify the difference of hydrogen's effects with different modalities of administration, each research group should scrutinize the difference of the effects between hydrogen gas, hydrogen water, and hydrogen-rich saline. This would uncover the best modality for each disease model, if any, and also the optimal hydrogen dose.

Table 1 summarizes disease categories for which the effects of hydrogen have been reported. Ohsawa and colleagues reported the hydrogen effect in cerebral infarction [1] and many subsequent studies also showed its effect in ischemia-reperfusion injuries including organ transplantations. Following the initial report by Ohsawa and colleagues, the specific hydroxyl radical scavenging effect of hydrogen has been repeatedly proposed in oxidative stress-mediated diseases including inflammatory diseases and metabolic diseases.

Table 2 shows the details of organs and diseases for which the effects of hydrogen have been reported. Table 2 is an update of our previous review article in 2012 [25]. We have now classified the organs and diseases into 31 categories and showed the effects in 166
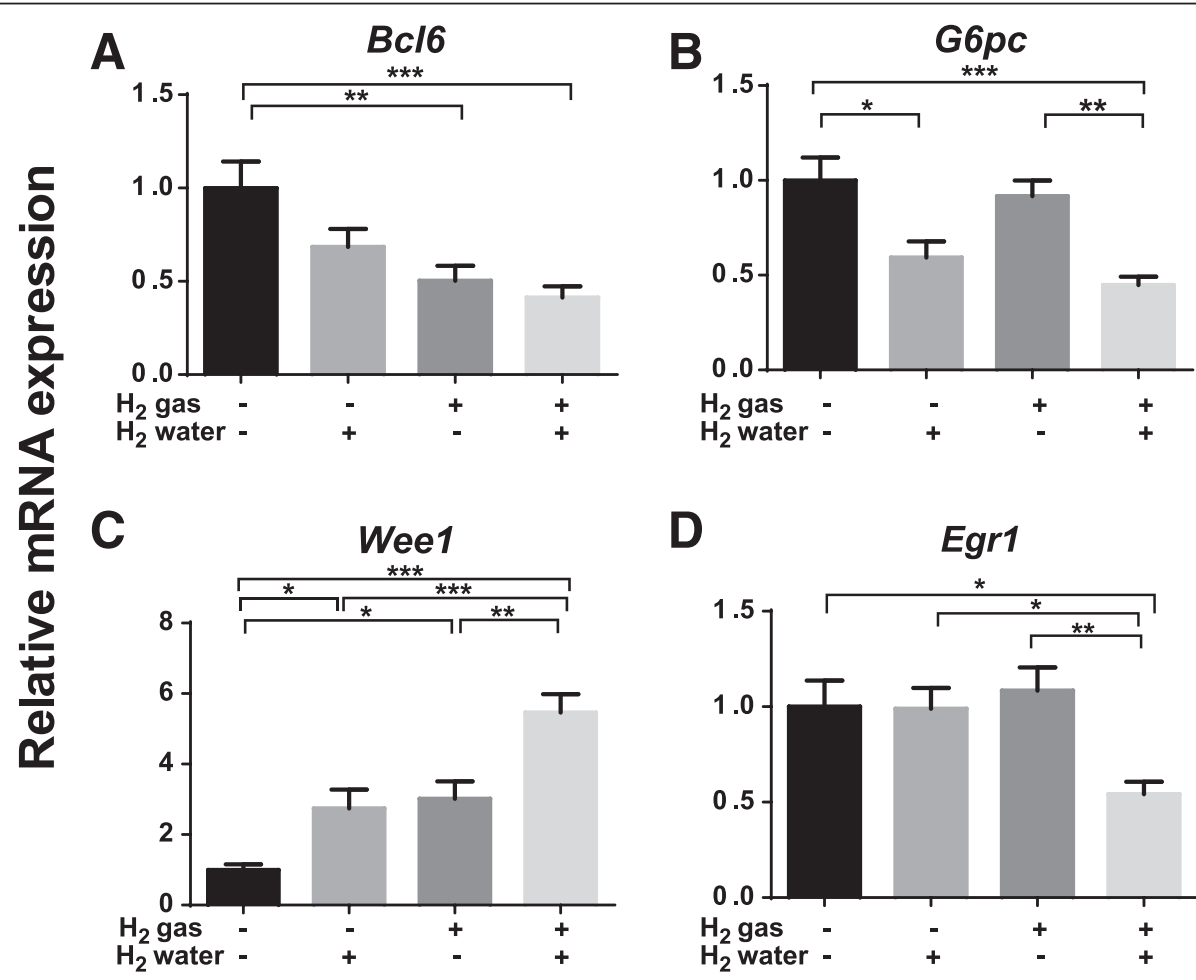

D

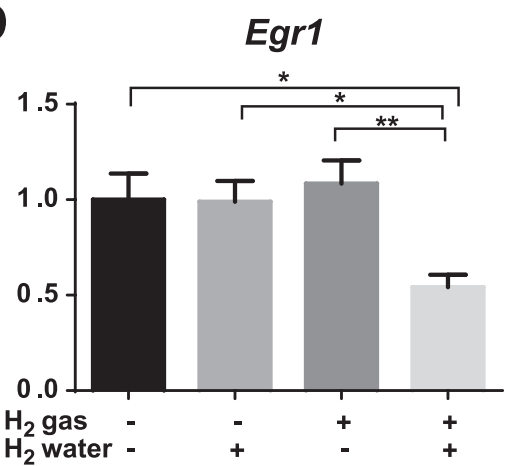

Fig. 2 Four groups of genes that show different responses to hydrogen gas and/or water [12] . a $B c / 6$ responds to hydrogen gas more than hydrogen water. $\mathbf{b}$ G6pc responds only to hydrogen water. c Weel responds to both hydrogen water and gas. d Egr1 responds only to simultaneous administration of hydrogen gas and water 
Table 1 Disease categories for which hydrogen exhibited beneficial effects

\begin{tabular}{lll}
\hline Pathophysiology & No. of articles & $\%$ \\
\hline Oxidative stress & 224 & 69.8 \\
(I/R injury & 80 & $24.9)$ \\
(Others & 144 & $44.9)$ \\
Inflammation & 66 & 20.6 \\
Metabolism & 20 & 6.2 \\
Others & 11 & 3.4 \\
\hline
\end{tabular}

I/R ischemia/reperfusion

disease models, human diseases, treatment-associated pathologies, and pathophysiological conditions of plants. Hydrogen is effective in essentially all organs, as well as in plants.

\section{Molecular mechanisms of the effects of hydrogen}

Collation of the 321 original articles reveals that most communications address the anti-oxidative stress, antiinflammatory, and anti-apoptotic effects. Specific scavenging activities of hydroxyl radical and peroxynitrite, however, cannot fully explain the anti-inflammatory and anti-apoptotic effects, which should involve a number of fine-tuned signaling pathways. We have shown that hydrogen suppresses signaling pathways in allergies [26] and inflammation [27] without directly scavenging reactive oxygen/nitrogen species. Signaling molecules that are modulated by hydrogen include Lyn [26, 28], Ras [29], MEK [29, 30], ERK [12, 24, 29-37], p38 [12, 16, 24, 27, 30, 32, 33, 35-41], JNK [13, 24, 27, 30, 32, 33, 35-38, 40, 42-47], ASK1 [27, 46], Akt [12, 29, 36, 37, 48, 49], GTPRac1 [36], iNOS [27, 34, 36, 50-52], Nox1 [36], NF-кB p65 or NF-kB [12, 14, 27, 35-38, 40, 41, 43, 49, 53-75], I $\mathrm{B} \alpha[27,40,41,54,60,62,69,73,76]$, STAT3 [65, 77, 78], NFATc1 [12, 36, 78], c-Fos [36], GSK-3 $\beta$ [48, 79], ROCK [80]. Activities and expressions of these molecules are modified by hydrogen. Master regulator(s) that drive these modifications remain to be elucidated.

The anti-oxidative stress effect of hydrogen was first reported to be conferred by direct elimination of hydroxyl radical and peroxynitrite. Subsequent studies indicate that hydrogen activates the Nrf2-Keap1 system. Hydrogen activates Nrf2 [36, 81-87] and its downstream heme oxygenase-1 (HO-1) [36, 51, 52, 65, 71, 81, 82, 84-93]. Kawamura and colleagues reported that hydrogen did not mitigate hyperoxic lung injury in Nrf2knockout mice [82]. Similarly, Ohsawa and colleagues reported that hydrogen enhanced mitochondrial functions and induced nuclear translocation of $\mathrm{Nrf} 2$ at the Symposium of Medical Molecular Hydrogen in 2012 and 2013. They proposed that hydrogen induces an adaptive response against oxidative stress, which is also known as a hormesis effect. These studies indicate that the effect of hydrogen is mediated by Nrf2, but the mechanisms of how Nrf2 is activated by hydrogen remain to be solved.

Another interesting mechanism is that hydrogen modulates miRNA expressions [64, 94]. Hydrogen regulates expressions of miR-9, miR-21, and miR-199, and modifies expressions of IKK- $\beta$, NF- $\kappa B$, and PDCD4 in LPSactivated retinal microglia cells [64]. Similarly, analysis of miRNA profiles of hippocampal neurons during $I / R$ injury revealed that hydrogen inhibits I/R-induced expression of the miR-200 family by reducing ROS production, which has led to suppression of cell death [94]. However, modulation of miRNA expression cannot solely explain all the biological effects mediated by hydrogen. In addition, mechanisms underlying modulated miRNA expressions remain to be elucidated.

Matsumoto and colleagues reported that oral intake of hydrogen water increased gastric expression and secretion of ghrelin and that the neuroprotective effect of hydrogen water was abolished by the ghrelin receptorantagonist and by the ghrelin secretion-antagonist [95]. As stated above, we have shown that hydrogen water, but not hydrogen gas, prevented development of Parkinson's disease in a rat model [11]. Prominent effect of oral hydrogen intake rather than hydrogen gas inhalation may be partly accounted for by gastric induction of ghrelin.

Recently, Ohta and colleagues showed at the 5th Symposium of Medical Molecular Hydrogen at Nagoya, Japan in 2015 that hydrogen influences a free radical chain reaction of unsaturated fatty acid on cell membrane and modifies its lipid peroxidation process. Furthermore, they demonstrated that air-oxidized phospholipid that was produced either in the presence or absence of hydrogen in vitro, gives rise to different intracellular signaling and gene expression profiles when added to the culture medium. They also showed that this aberrant oxidization of phospholipid was observed with a low concentration of hydrogen (at least $1.3 \%$ ), suggesting that the biological effects of hydrogen could be explained by the aberrant oxidation of phospholipid under hydrogen exposure. Among the many molecules that are altered by hydrogen, most are predicted to be passengers (downstream regulators) that are modulated secondarily to a change in a driver (master regulator). The best way to identify the master regulator is to prove the effect of hydrogen in an in vitro system. Although, to our knowledge, the study on lipid peroxidation has not yet been published, the free radical chain reaction for lipid peroxidation might be the second master regulator of hydrogen next to the radical scavenging effect. We are also analyzing other novel molecules as possible master regulators of hydrogen (in preparation). Taken together, hydrogen is likely to have multiple master regulators, which drive a diverse array of downstream 
Table 2 Disease models, human diseases, treatment-associated pathologies, and pathophysiological conditions of plants (321 original articles published in English) for which the effects of hydrogen have been reported from 2007 to June 2015

\begin{tabular}{|c|c|}
\hline Diseases and conditions & References \\
\hline \multicolumn{2}{|l|}{ Brain } \\
\hline \multicolumn{2}{|l|}{ Cerebrovascular diseases (CVD) } \\
\hline Cerebral I/R injury & {$[1,10,56,83,94,99-109]$} \\
\hline Hypertensive stroke & [110] \\
\hline Brain injury secondary to intracerebral hemorrhage & [28] \\
\hline Subarachnoid hemorrhage & {$[48,61,66,73,111-113]$} \\
\hline \multicolumn{2}{|l|}{ Brain injury other than CVD } \\
\hline Traumatic brain injury & [114-118] \\
\hline Deep hypothermic circulatory arrest-induced brain damage & {$[57]$} \\
\hline \multicolumn{2}{|l|}{ Neurodegenerative diseases } \\
\hline Parkinson's disease & {$[11,95-97,119]$} \\
\hline Alzheimer's disease & {$[43,120]$} \\
\hline \multicolumn{2}{|l|}{ Others } \\
\hline Restraint-induced dementia & {$[121]$} \\
\hline Senile dementia in senescence-accelerated mice & {$[122]$} \\
\hline LPS-induced neuroinflammation & {$[81,123]$} \\
\hline Oxidative stress-induced neuronal cell damage & {$[124,125]$} \\
\hline \multicolumn{2}{|l|}{ Spinal Cord and peripheral nerve } \\
\hline Spinal cord I/R injury & {$[126,127]$} \\
\hline Spinal cord injury & {$[77,128]$} \\
\hline Neuropathic pain & {$[39,92,129,130]$} \\
\hline Hyperalgesia & {$[79,131,132]$} \\
\hline \multicolumn{2}{|l|}{ Eye } \\
\hline Retinal I/R injury & {$[133,134]$} \\
\hline Diabetic retinopathy & {$[135,136]$} \\
\hline Hyperoxia-induced retinopathy & {$[137]$} \\
\hline Light-induced retinopathy & {$[138,139]$} \\
\hline Glutamine-induced retinopathy & {$[50]$} \\
\hline S-nitroso-N-acetylpenicillamine-induced retinopathy & {$[140]$} \\
\hline Optic nerve crush & [141] \\
\hline Selenite-induced cataract & {$[142]$} \\
\hline Corneal alkali-burn & {$[55]$} \\
\hline Anti-inflammatory effects on LPS-activated retinal microglia cells & {$[64]$} \\
\hline \multicolumn{2}{|l|}{ Ear } \\
\hline Hearing loss & {$[143-148]$} \\
\hline Cisplatin-induced ototoxicity & {$[149,150]$} \\
\hline Ouabain-induced ototoxicity & {$[151]$} \\
\hline \multicolumn{2}{|l|}{ Oral Cavity } \\
\hline Periodontitis & {$[32]$} \\
\hline Periodontal oxidative damage & [152] \\
\hline \multicolumn{2}{|l|}{ Lung } \\
\hline Lung I/R injury & {$[153,154]$} \\
\hline Oxygen-induced lung injury & {$[82,155,156]$} \\
\hline
\end{tabular}


Table 2 Disease models, human diseases, treatment-associated pathologies, and pathophysiological conditions of plants (321 original articles published in English) for which the effects of hydrogen have been reported from 2007 to June 2015 (Continued)

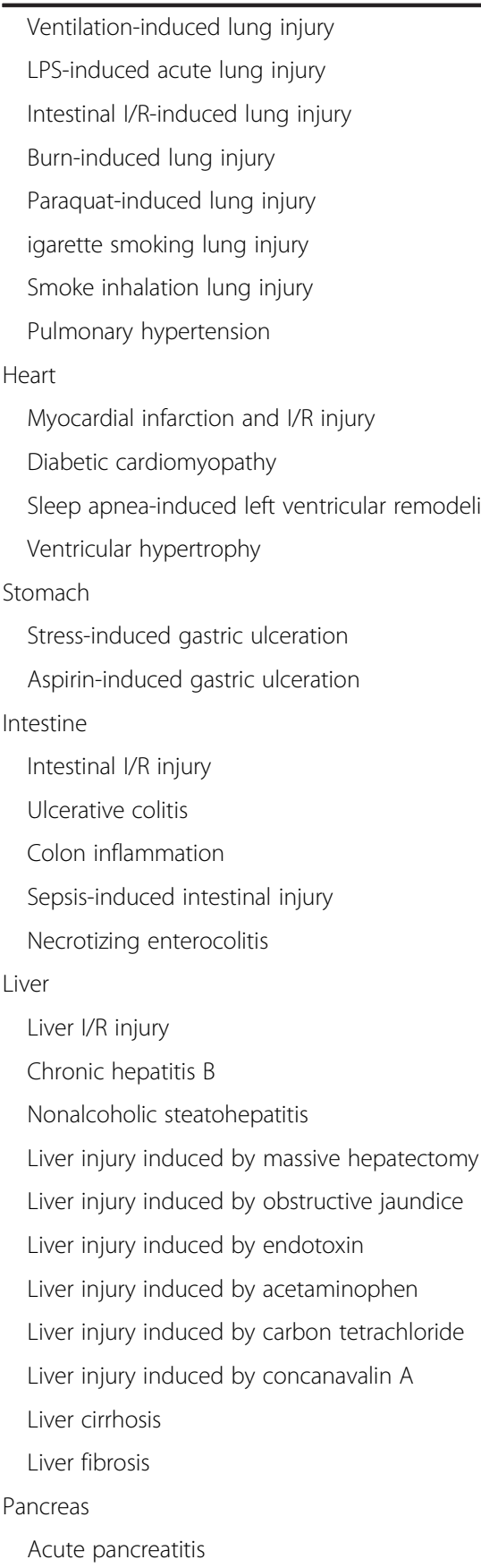

Peritoneum

Kidney

Renal I/R injury 
Table 2 Disease models, human diseases, treatment-associated pathologies, and pathophysiological conditions of plants (321 original articles published in English) for which the effects of hydrogen have been reported from 2007 to June 2015 (Continued)

Inhibition of AGEs production

Renal calcium deposition

Bladder

Interstitial cystitis

Reproductive organ

Testicular I/R injury

Erectile dysfunction

Nicotine-induced testicular oxidative stress

Cigarette smoke-induced testicular damage

Skin

I/R injury

UV-induced skin injury

Acute erythematous skin disease

Atopic dermatitis

Psoriasis

Pressure ulcer

Burn

Arsenic toxicity

Bone and Joint

Rheumatoid arthritis

Osteoporosis

Bone loss induced by microgravity

TNFa-induced osteoblast injury

NO-induced cartilage toxicity

Skeletal Muscle and soft tissue

I/R injury in skeletal muscle

Inflammatory and mitochondrial myopathies

Muscle fatigue

Sports-related soft tissue injury

Blood vessel

Atherosclerosis

AGEs-induced blood vessel damage

Neointimal hyperplasia

Hyperplasia in arterialized vein graft

Vascular dysfunction

Vascular endothelial function

Blood and Bone Marrow

Aplastic anemia

Maintenance of multipotential stroma/mesenchymal stem cells

Neutrophil function

Inhibition of collagen-induced platelet aggregation

Improvement of blood fluidity

Metabolism

Diabetes mellitus
[199]

[200]

$[46,207]$

$[45,208-211]$

[212]

$[213,214]$

[215]

[216]

$[49,70]$

[217]

$[218,219]$

$[36,62]$

[34]

[220]

[221]

$[58,59,85,226,227]$

[228]

[29]

[229]

[60]

[230]

[232] 
Table 2 Disease models, human diseases, treatment-associated pathologies, and pathophysiological conditions of plants (321 original articles published in English) for which the effects of hydrogen have been reported from 2007 to June 2015 (Continued)

\begin{tabular}{|c|c|}
\hline Hyperlipidemia & {$[44,242-244]$} \\
\hline Metabolic syndrome & {$[245-247]$} \\
\hline Metabolic process-related gene expression & [248] \\
\hline Oxidized low density lipoprotein-induced cell toxicity & [54] \\
\hline Serum alkalinization & [249] \\
\hline Exercise-induced metabolic acidosis & [250] \\
\hline \multicolumn{2}{|l|}{ Inflammation/Allergy } \\
\hline Sepsis & {$[41,86,251-255]$} \\
\hline LPS/IFNy-induced NO production & [27] \\
\hline LPS-induced inflammatory response & [90] \\
\hline LPS-induced vascular permeability & {$[80,256]$} \\
\hline Zymosan-induced inflammation & [257] \\
\hline Carrageenan-induced paw edema & [258] \\
\hline Inflammatory response of cardiopulmonary bypass & [259] \\
\hline Type I allergy & [26] \\
\hline Asthma & [63] \\
\hline \multicolumn{2}{|l|}{ Perinatal Disorders } \\
\hline Neonatal cerebral hypoxia & {$[260-263]$} \\
\hline LPS-induced fetal lung injury & [15] \\
\hline Preeclampsia & {$[264,265]$} \\
\hline \multicolumn{2}{|l|}{ Cancer } \\
\hline Growth of tongue carcinoma cells & [266] \\
\hline Fe-NTA-induced nephrotoxicity and tumor progression & [65] \\
\hline Radiation-induced thymic lymphoma & [267] \\
\hline Tumor angiogenesis & [268] \\
\hline Enhancement of 5-FU antitumor efficacy & [269] \\
\hline \multicolumn{2}{|l|}{ Radiation } \\
\hline Cardiac damage & {$[270]$} \\
\hline Lung damage & [271] \\
\hline Testicular damage & [272] \\
\hline Skin damage & {$[273,274]$} \\
\hline Germ, hematopoietic and other cell damage & {$[275-280]$} \\
\hline Radiation-induced adverse effects & [281] \\
\hline Radiation-induced immune dysfunction & [282] \\
\hline \multicolumn{2}{|l|}{ Intoxication } \\
\hline Carbon monoxide & [283-286] \\
\hline Sevoflurane & {$[287,288]$} \\
\hline Doxorubicin-induced heart failure & [289] \\
\hline Melamine-induced urinary stone & [290] \\
\hline Chlorpyrifos-induced neurotoxicity & [291] \\
\hline \multicolumn{2}{|l|}{ Transplantation } \\
\hline Heart & {$[52,292-294]$} \\
\hline Lung & {$[33,88,295-299]$} \\
\hline Kidney & {$[30,51]$} \\
\hline
\end{tabular}


Table 2 Disease models, human diseases, treatment-associated pathologies, and pathophysiological conditions of plants (321 original articles published in English) for which the effects of hydrogen have been reported from 2007 to June 2015 (Continued)

\begin{tabular}{|c|c|}
\hline Intestine & {$[89,300,301]$} \\
\hline Pancreas & {$[302]$} \\
\hline Osteochondral grafts & [303] \\
\hline Acute GVHD & {$[304,305]$} \\
\hline \multicolumn{2}{|l|}{ Resuscitation } \\
\hline Cardiac arrest & {$[306,307]$} \\
\hline Hemorrhagic shock & {$[75,308,309]$} \\
\hline \multicolumn{2}{|l|}{ Dialysis } \\
\hline Hemodialysis & [310-313] \\
\hline Peritoneal dialysis & {$[314,315]$} \\
\hline \multicolumn{2}{|l|}{ Others } \\
\hline Lifespan extension & {$[316]$} \\
\hline Sperm motility & {$[317]$} \\
\hline Decompression sickness & [318] \\
\hline Genotoxicity and mutagenicity & [319] \\
\hline \multicolumn{2}{|l|}{ Plant } \\
\hline Root organogenesis & {$[91,320]$} \\
\hline Salt tolerance & {$[321,322]$} \\
\hline Postharvest ripening & [323] \\
\hline Stomatal closure & [324] \\
\hline Radish sprout tolerance to UVA & {$[325]$} \\
\hline High light stress & {$[326]$} \\
\hline Phytohormone signaling and stress responses & {$[327]$} \\
\hline Tolerance to paraquat-induced oxidative stress & {$[328]$} \\
\hline Cadmium toxicity & {$[329,330]$} \\
\hline Mercury toxicity & {$[331]$} \\
\hline
\end{tabular}

regulators and achieve beneficial biological effects against oxidative stress, inflammation, apoptosis, and dysmetabolism to name a few (Fig. 3).

These studies all point to the notion that hydrogen modulates intracellular signal transduction systems and regulates the downstream gene expressions to mitigate disease processes. In general, biologically active substances that modulate signaling molecules have both beneficial and noxious effects on our bodies. Hydrogen may also have undisclosed toxic effects, although none have been reported to date to the best of our knowledge. Understanding the exact molecular mechanisms of the effects of hydrogen will elucidate its master regulator(s) and clarify the pros and cons of hydrogen therapy, which will also potentially lead to the development of another therapeutic modality to modulate the master regulator(s). We summarized in Table 3 original articles that addressed biological effects and in vivo kinetics of hydrogen, which were not directly relevant to disease models or human diseases. It is essential to elucidate detailed pharmacokinetics of hydrogen in vivo from the viewpoint of clinical application of hydrogen, although we have accumulated vast knowledge about the effects and not the kinetics of hydrogen in disease models and human diseases. Through these analyses, promising outcomes are expected for more effective administration regimen of hydrogen therapy.

\section{Clinical studies of molecular hydrogen}

As stated in the introduction, the number of clinical trials has been increasing since 2011. About half of human studies have been conducted in Japan. Dependable studies recruiting more than ten patients or employing double-blind studies are summarized in Table 4.

Features shared in these clinical studies are that hydrogen exhibits statistically significant effects in patients but the effects are usually not as conspicuous as those observed in rodent models. These can be accounted for by i) the difference in species, ii) technical difficulty in preparing a high concentration of hydrogen water every day 


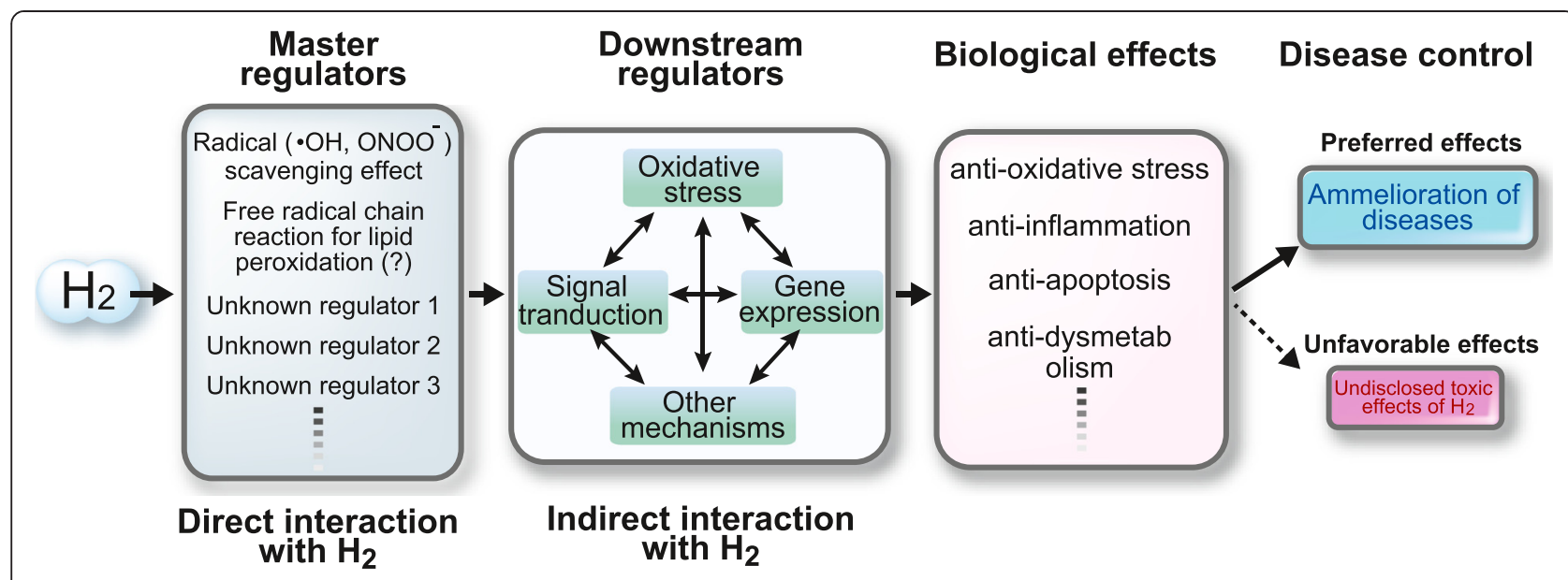

Fig. 3 Schematic summary of molecular mechanisms of hydrogen

for the patients, and iii) the difference between acute and chronic diseases. Further large-scale and long-term clinical studies are expected to prove the effects of hydrogen in humans.

Table 5 shows clinical studies currently registered in Japan. Researchers in Juntendo University have started a large-scale clinical trial of Parkinson's disease after they have shown the effects of molecular hydrogen in a small number of patients in a short duration [96]. Being prompted by the prominent effects of hydrogen for mouse models with ischemia reperfusion injuries, clinical trials for acute post cardiac arrest syndrome and myocardial infarction have started at Keio University. Similarly, a clinical trial for cerebral infarction has started at the National Defense Medical College.

\section{Conclusions}

The number of original articles showing the effects of hydrogen are increasing yearly after 2007, and an extensive review of these articles are getting more and more difficult. Some of these articles, however, are a repetition of previous studies with insignificant novel findings. We suppose that almost all disease models and almost all modalities by which hydrogen is administered have been already examined. Large-scale controlled human studies and elucidation of molecular mechanisms underlying the effects of hydrogen are the next steps that must be pursued.

A dose-response effect of hydrogen is observed in drinking hydrogen-rich water [94, 97]. A similar doseresponse effect is also observed in inhaled hydrogen gas $[1,17,98]$. However, when hydrogen concentrations in drinking water and in inhaled gas are compared, there is no dose-response effect. Hydrogen-rich water generally shows a more prominent effect than hydrogen gas, although the amount of hydrogen taken up by hydrogen water is $\sim 100$ times less than that given by hydrogen gas [11]. Gastric secretion of ghrelin may partly account for

Table 3 Original articles showing physiological effects and in vivo kinetics of hydrogen

\begin{tabular}{|c|c|}
\hline Biological effects and in vivo kinetics of hydrogen & References \\
\hline Superoxide formation in brain slices in mice & [332] \\
\hline Gene expression profiles and signal transduction pathways evaluated by DNA microarray and RNA-seq in rodents & {$[33]^{a},[12],[118]^{a},[248]^{a}$} \\
\hline Comparison of intermittent and continuous administration of hydrogen gas in rats & {$[11]^{a}$} \\
\hline Safety of hydrogen inhalation in patients with cerebral ischemia & [333] \\
\hline A convenient method to estimate the concentration of hydrogen in water & [334] \\
\hline Hydrogen consumption in human body after hydrogen administration & {$[335,336]$} \\
\hline Ghrelin induction and secretion by hydrogen-dissolved water in mice & {$[95]^{\mathrm{a}}$} \\
\hline Additive effects of hydrogen and NO & {$[20,158]^{a}$} \\
\hline In vivo kinetics of hydrogen after hydrogen administration in rodents & {$[12,337]$} \\
\hline Lack of reactivity of hydrogen with peroxynitrite & {$[338]$} \\
\hline Antioxidant activity of nano-bubble hydrogen-dissolved water & {$[339]$} \\
\hline Additive effects of hydrogen gas and hydrogen-rich water & [12] \\
\hline
\end{tabular}

${ }^{\mathrm{a}}$ These articles are also listed in Table 2 
Table 4 Clinical trials published as of June, 2015

\begin{tabular}{|c|c|c|c|c|c|}
\hline Authors/Year & Disease & $\begin{array}{l}\text { Sample } \\
\text { size }\end{array}$ & $\begin{array}{l}\text { Open-label (OL), } \\
\text { double-blind (DB), or } \\
\text { single-blind (SB) }\end{array}$ & $\begin{array}{l}\text { Hydrogen } \\
\text { administration }\end{array}$ & Summary of the outcome \\
\hline $\begin{array}{l}\text { Kajiyama et al. } \\
{[236] / 2008}\end{array}$ & $\begin{array}{l}\text { Diabetes mellitus } \\
\text { type II }\end{array}$ & 30 & DB & Water & $\begin{array}{l}\text { Improvement of fractions of low-density lipoprotein } \\
\text { (LDL)-cholesterol and a glucose tolerance test. }\end{array}$ \\
\hline $\begin{array}{l}\text { Nakao et al. } \\
{[245] / 2010}\end{array}$ & $\begin{array}{l}\text { Metabolic } \\
\text { syndrome }\end{array}$ & 20 & $\mathrm{OL}$ & Water & $\begin{array}{l}\text { Improvement of urinary markers for oxidative stress such } \\
\text { as SOD and TBARS, and increase of high-density lipopro- } \\
\text { tein (HDL)-cholesterol. }\end{array}$ \\
\hline $\begin{array}{l}\text { Nakayama et al. } \\
{[311] / 2010}\end{array}$ & Chronic renal failure & 29 & $\mathrm{OL}$ & Dialysis & $\begin{array}{l}\text { Amelioration of hypertension and improvement of markers } \\
\text { for oxidative stress and inflammation. }\end{array}$ \\
\hline \multirow[t]{2}{*}{$\begin{array}{l}\text { Ito et al. } \\
\text { [223]/2011 }\end{array}$} & \multirow[t]{2}{*}{$\begin{array}{l}\text { Inflammatory and } \\
\text { mitochondrial } \\
\text { myopathies }\end{array}$} & \multirow[t]{2}{*}{31} & \multirow[t]{2}{*}{ OL/DB } & \multirow[t]{2}{*}{ Water } & $\begin{array}{l}\text { OL: Improvement of the serum lactate/pyruvate ratio in } \\
\text { mitochondrial myopathies and the serum matrix } \\
\text { metalloproteinse-3 level in polymyositis/dermatomyositis. }\end{array}$ \\
\hline & & & & & DB: Improvement of the serum lactate. \\
\hline \multirow[t]{2}{*}{$\begin{array}{l}\text { Kang et al. } \\
{[281] / 2011}\end{array}$} & \multirow{2}{*}{$\begin{array}{l}\text { Radiation-induced } \\
\text { adverse effects for } \\
\text { liver tumors }\end{array}$} & \multirow[t]{2}{*}{49} & \multirow[t]{2}{*}{$\mathrm{OL}$} & \multirow[t]{2}{*}{ Water } & $\begin{array}{l}\text { Improvement of quality of life (QOL) scores during } \\
\text { radiotherapy. }\end{array}$ \\
\hline & & & & & $\begin{array}{l}\text { Reduction of blood reactive oxygen metabolites and } \\
\text { maintenance of blood oxidation potential. }\end{array}$ \\
\hline \multirow[t]{2}{*}{$\begin{array}{l}\text { Ishibashi et al. } \\
{[218] / 2012}\end{array}$} & \multirow[t]{2}{*}{ Rheumatoid arthritis } & \multirow[t]{2}{*}{20} & \multirow[t]{2}{*}{$\mathrm{OL}$} & \multirow[t]{2}{*}{ Water } & $\begin{array}{l}\text { Improvement of disease activity score for rheumatoid } \\
\text { arthritis (DAS28). }\end{array}$ \\
\hline & & & & & Decrease of urinary 8-OHdG. \\
\hline $\begin{array}{l}\text { Aoki et al. } \\
{[224] / 2012}\end{array}$ & Muscle fatigue & 10 & $\mathrm{DB}$ & Water & Improvement of muscle fatigue in young athletes \\
\hline $\begin{array}{l}\text { Li et al. } \\
{[216] / 2013}\end{array}$ & Pressure skin ulcer & 22 & $\mathrm{OL}$ & Water & $\begin{array}{l}\text { Wound size reduction and early recovery from skin } \\
\text { pressure ulcer. }\end{array}$ \\
\hline \multirow{2}{*}{$\begin{array}{l}\text { Matsumoto et al. } \\
{[201] / 2013}\end{array}$} & \multirow{2}{*}{ Interstitial cystitis } & \multirow[t]{2}{*}{30} & \multirow[t]{2}{*}{ DB } & \multirow[t]{2}{*}{ Water } & No significant effect on symptoms. \\
\hline & & & & & Reduction of the bladder pain score in $11 \%$ of patients. \\
\hline \multirow{2}{*}{$\begin{array}{l}\text { Nagatani et al. } \\
{[106] / 2013}\end{array}$} & \multirow[t]{2}{*}{ Cerebral ischemia } & \multirow[t]{2}{*}{38} & \multirow[t]{2}{*}{$\mathrm{OL}$} & \multirow{2}{*}{$\begin{array}{l}\text { Intravenous } \\
\text { infusion }\end{array}$} & Confirmation of safety of intravenous $\mathrm{H}_{2}$ infusion. \\
\hline & & & & & $\begin{array}{l}\text { Decrease of MDA-LDL, a serum marker for oxidative } \\
\text { stress, in a subset of patients. }\end{array}$ \\
\hline $\begin{array}{l}\text { Shin et al. } \\
{[45] / 2013}\end{array}$ & $\begin{array}{l}\text { UV-induced skin } \\
\text { injury }\end{array}$ & 28 & $\mathrm{OL}$ & Gas & $\begin{array}{l}\text { Prevention and modulation of UV-induced skin } \\
\text { inflammation, intrinsic skin aging, and photo aging } \\
\text { process through reduction of MMP-1, IL-6, and IL-1b } \\
\text { mRNA expression. }\end{array}$ \\
\hline $\begin{array}{l}\text { Song et al. } \\
{[243] / 2013}\end{array}$ & Hyperlipidemia & 20 & $\mathrm{OL}$ & Water & $\begin{array}{l}\text { Decrease of total serum cholesterol, LDL-cholesterol, } \\
\text { apolipoprotein (apo) B100, and apoE }\end{array}$ \\
\hline $\begin{array}{l}\text { Xia et al. } \\
\text { [179]/2013 }\end{array}$ & Chronic hepatitis B & 60 & $\mathrm{DB}$ & Water & Attenuation of oxidative stress \\
\hline $\begin{array}{l}\text { Yoritaka et al. } \\
{[96] / 2013}\end{array}$ & Parkinson disease & 17 & $\mathrm{DB}$ & Water & $\begin{array}{l}\text { Improvement of Total Unified Parkinson's Disease Rating } \\
\text { Scale (UPDRS) and exacerbation after termination of } \mathrm{H}_{2} \\
\text { water. }\end{array}$ \\
\hline \multirow{2}{*}{$\begin{array}{l}\text { Ishibashi et al. } \\
\text { [219]/2014 }\end{array}$} & \multirow[t]{2}{*}{ Rheumatoid arthritis } & \multirow[t]{2}{*}{24} & \multirow[t]{2}{*}{ DB } & \multirow{2}{*}{$\begin{array}{l}\text { Intravenous } \\
\text { saline infusion }\end{array}$} & Improvement of DAS28. \\
\hline & & & & & Decrease of serum IL-6, MMP3, CRP, and urinary 8-OHdG. \\
\hline \multirow{2}{*}{$\begin{array}{l}\text { Ostojic et al. } \\
{[225] / 2014}\end{array}$} & \multirow{2}{*}{$\begin{array}{l}\text { Sports-related soft } \\
\text { tissue injury }\end{array}$} & \multirow[t]{2}{*}{36} & SB & $\mathrm{H} 2$-rich tablets & Decrease of plasma viscosity. \\
\hline & & & & packs & Faster recovery from soft tissue injury. \\
\hline $\begin{array}{l}\text { Ostojic et al. } \\
{[250] / 2014}\end{array}$ & $\begin{array}{l}\text { Exercise-induced } \\
\text { metabolic acidosis }\end{array}$ & 52 & $\mathrm{DB}$ & Water & Increased blood alkalinity in physically active men. \\
\hline $\begin{array}{l}\text { Sakai et al. } \\
\text { [230]/2014 }\end{array}$ & $\begin{array}{l}\text { Vascular endothelial } \\
\text { function. }\end{array}$ & 34 & $\mathrm{DB}$ & Water & $\begin{array}{l}\text { Increased flow-mediated dilation of branchial artery, } \\
\text { suggesting that } \mathrm{H}_{2} \text { can serve as a modulator of } \\
\text { vasomotor function of vasculature. }\end{array}$ \\
\hline $\begin{array}{l}\text { Song et al. } \\
{[244] / 2015}\end{array}$ & Hyperlipidemia & 68 & DB & Water & $\begin{array}{l}\text { Down-regulation of plasma levels of total cholesterol, and } \\
\mathrm{LDL}-\mathrm{cholesterol} \text {, followed by increased plasma pre- } \beta \text {-HDL, } \\
\text { apoM, and decreased plasma oxidized-LDL, apoB100. }\end{array}$ \\
\hline
\end{tabular}


Table 5 Clinical trials registered in Japan as of June, 2015

\begin{tabular}{|c|c|c|c|}
\hline Date & Disease & Affiliation & Status \\
\hline $7 / 16 / 2008$ & Interstitial cystitis & Koshinkai Hosp. & Finished[201] \\
\hline $8 / 21 / 2008$ & $\begin{array}{l}\text { Impaired glucose tolerance and } \\
\text { impaired fasting glycaemia }\end{array}$ & $\begin{array}{l}\text { Digestive tract internal medicine, Kyoto } \\
\text { Prefectural University of Medicine }\end{array}$ & Finished [236]. \\
\hline $7 / 17 / 2009$ & Mild cognitive impairment & Neuropsychiatry, Tsukuba Univ. & Finished \\
\hline $1 / 11 / 2011$ & Chronic hemodialysis & Nephrology, Fukushima Medical University & Trial in progress \\
\hline $6 / 2 / 2011$ & Acute cerebral infarction & Neurosurgery, Self Defense Medical College & Calling for participants[106] \\
\hline 9/30/2011 & Normal adults & Faculty of Health Sciences, Kyorin Univ. & Finished \\
\hline $12 / 4 / 2011$ & Acute myocardial infarction & Cardiology, Keio Univ. & Calling for participants \\
\hline $3 / 14 / 2012$ & Parkinson's disease & Neurology, Juntendo Univ. & Finished [96] \\
\hline 10/16/2012 & $\begin{array}{l}\text { Multiple system atrophy, Progressive } \\
\text { supranuclear palsy }\end{array}$ & Neurology, Juntendo Univ. & Trial in progress \\
\hline 2/13/2013 & Parkinson's disease & Neurology, Juntendo Univ. & Calling for participants \\
\hline $5 / 1 / 2013$ & Chronic obstructive pulmonary disease & Respiratory Medicine, Juntendo Univ. & Trial in progress \\
\hline $5 / 20 / 2013$ & Hepatitis and liver cirrhosis & Gastroenterology and Hepatology, Okayama Univ. & In preparation \\
\hline $11 / 22 / 2013$ & Post cardiac arrest syndrome & Emergency and Critical care medicine, Keio Univ. & Calling for participants \\
\hline $2 / 22 / 2014$ & Eye disease & Ophthalmology, Nippon Medical school & Finished \\
\hline $7 / 1 / 2014$ & Acute myocardial infarction & Cardiology, National Center for Global Health and Medicine & Calling for participants \\
\hline $7 / 29 / 2014$ & Subarachnoid hemorrhage & Neurosurgery, Self Defense Medical College & Calling for participants [113] \\
\hline $8 / 1 / 2014$ & Lung transplantation & General thoracic surgery, Osaka Univ. & Calling for participants \\
\hline $10 / 27 / 2014$ & Retinal artery occlusion & Ophthalmology, Nippon Medical school & Calling for participants \\
\hline $7 / 3 / 2015$ & Type 2 diabetes mellitus & Tokyo Metropolitan Institute of Gerontology & Calling for participants \\
\hline
\end{tabular}

The department names are shown if they are available in the UMIN clinical trial database

this difference [95]. Another factor that accounts for the effects of hydrogen is the temporal profile of hydrogen administration. Intermittent inhalation, but not continuous inhalation, of hydrogen is protective against a rat model of Parkinson's disease, which is against a doseresponsiveness of hydrogen [11]. The prominent effects of molecular hydrogen in a variety of disease models, human diseases, treatment-associated pathologies, and pathophysiological conditions of plants have been disclosed in these 8 years, but unsolved conundrums still challenge us.

\section{Competing interests}

We have no competing interest to disclose.

\section{Authors' contributions}

$\mathrm{Ml}^{1}$ collated and scrutinized all hydrogen papers. SS, $\mathrm{Ml}^{2}, \mathrm{Ml}^{3}$, and $\mathrm{MH}$ made critical comments on hydrogen papers. $\mathrm{Ml}^{1}$ and $\mathrm{KO}$ wrote the paper. All authors read and approved the final manuscript.

\section{Acknowledgements}

Works done in our laboratories were supported by Grants-in-Aid from the Ministry of Education, Culture, Sports, Science and Technology of Japan (MEXT), the Ministry of Health, Labor and Welfare (MHLW) of Japan, the Japan Agency for Medical Research and Development (AMED), and Chubu University Grants A and B.

\section{Author details}

${ }^{1}$ Department of Biomedical Sciences, College of Life and Health Sciences, Chubu University, 1200 Matsumoto-cho, Kasugai 487-8501, Japan. ²Division of Neurogenetics, Center for Neurological Diseases and Cancer, Nagoya
University Graduate School of Medicine, 65 TsurumaiShowa-ku, Nagoya 466-8550, Japan. ${ }^{3}$ Research Team for Mechanism of Aging, Tokyo Metropolitan Institute of Gerontology, 35-2 Sakae-cho, Itabashi, Tokyo 173-0015, Japan. ${ }^{4}$ Department of Pathophysiological Laboratory Sciences, Nagoya University Graduate School of Medicine, 1-1-20 Daiko-Minami, Higashi-ku, Nagoya 461-8673, Japan.

Received: 20 July 2015 Accepted: 9 October 2015

Published online: 19 October 2015

\section{References}

1. Ohsawa I, Ishikawa M, Takahashi K, Watanabe M, Nishimaki K, Yamagata K, et al. Hydrogen acts as a therapeutic antioxidant by selectively reducing cytotoxic oxygen radicals. Nat Med. 2007;13(6):688-94. doi:10.1038/nm1577.

2. Dole M, Wilson FR, Fife WP. Hyperbaric hydrogen therapy: a possible treatment for cancer. Science. 1975;190(4210):152-4.

3. Roberts BJ, Fife WP, Corbett TH, Schabel Jr FM. Response of five established solid transplantable mouse tumors and one mouse leukemia to hyperbaric hydrogen. Cancer Treat Rep. 1978;62(7):1077-9.

4. Abraini JH, Gardette-Chauffour MC, Martinez E, Rostain JC, Lemaire C. Psychophysiological reactions in humans during an open sea dive to $500 \mathrm{~m}$ with a hydrogen-helium-oxygen mixture. J Appl Physiol. 1994;76(3):1113-8.

5. Gharib B, Hanna S, Abdallahi OM, Lepidi H, Gardette B, De Reggi M. Anti-inflammatory properties of molecular hydrogen: investigation on parasite-induced liver inflammation. C R Acad Sci III. 2001;324(8):719-24.

6. Shirahata S, Kabayama S, Nakano M, Miura T, Kusumoto K, Gotoh M, et al. Electrolyzed-reduced water scavenges active oxygen species and protects DNA from oxidative damage. Biochem Biophys Res Commun. 1997;234(1):269-74. doi:10.1006/bbrc.1997.6622.

7. Li Y, Nishimura T, Teruya K, Maki T, Komatsu T, Hamasaki T, et al. Protective mechanism of reduced water against alloxan-induced pancreatic beta-cell damage: Scavenging effect against reactive oxygen species. Cytotechnology. 2002;40(1-3):139-49. doi:10.1023/A:1023936421448. 
8. Huang KC, Yang CC, Lee KT, Chien CT. Reduced hemodialysis-induced oxidative stress in end-stage renal disease patients by electrolyzed reduced water. Kidney Int. 2003;64(2):704-14. doi:10.1046/j.1523-1755.2003.00118.x.

9. Yanagihara T, Arai K, Miyamae K, Sato B, Shudo T, Yamada M, et al. Electrolyzed hydrogen-saturated water for drinking use elicits an antioxidative effect: a feeding test with rats. Biosci Biotechnol Biochem. 2005;69(10):1985-7.

10. Huang G, Zhou J, Zhan W, Xiong Y, Hu C, Li X, et al. The neuroprotective effects of intraperitoneal injection of hydrogen in rabbits with cardiac arrest. Resuscitation. 2013;84(5):690-5. doi:10.1016/j.resuscitation.2012.10.018.

11. Ito $M$, Hirayama M, Yamai $K$, Goto S, Ito M, Ichihara $M$, et al. Drinking hydrogen water and intermittent hydrogen gas exposure, but not lactulose or continuous hydrogen gas exposure, prevent 6-hydorxydopamineinduced Parkinson's disease in rats. Med Gas Res. 2012;2(1):15. doi:10.1186/2045-9912-2-15

12. Sobue S, Yamai K, Ito M, Ohno K, Ito M, Iwamoto T, et al. Simultaneous oral and inhalational intake of molecular hydrogen additively suppresses signaling pathways in rodents. Mol Cell Biochem. 2015;403(1-2):231-41. doi:10.1007/s11010-015-2353-y.

13. Qiu X, Li H, Tang H, Jin Y, Li W, Sun Y, et al. Hydrogen inhalation ameliorates lipopolysaccharide-induced acute lung injury in mice. Int Immunopharmacol. 2011;11(12):2130-7. doi:10.1016/j.intimp.2011.09.007.

14. Xie K, Yu Y, Huang Y, Zheng L, Li J, Chen H, et al. Molecular hydrogen ameliorates lipopolysaccharide-induced acute lung injury in mice through reducing inflammation and apoptosis. Shock. 2012;37(5):548-55. doi:10.1097/SHK.0b013e31824ddc81.

15. Hattori Y, Kotani T, Tsuda H, Mano Y, Tu L, Li H, et al. Maternal molecular hydrogen treatment attenuates lipopolysaccharide-induced rat fetal lung injury. Free Radic Res. 2015;49:1026-37. doi:10.3109/10715762.2015.1038257.

16. Zhang Y, Liu Y, Zhang J. Saturated hydrogen saline attenuates endotoxininduced lung dysfunction. J Surg Res. 2015;198:41-9. doi:10.1016/ j.jss.2015.04.055.

17. Hayashida K, Sano M, Ohsawa I, Shinmura K, Tamaki K, Kimura K, et al. Inhalation of hydrogen gas reduces infarct size in the rat model of myocardial ischemia-reperfusion injury. Biochem Biophys Res Commun. 2008:373(1):30-5. doi:10.1016/j.bbrc.2008.05.165.

18. Sakai K, Cho S, Shibata I, Yoshitomi O, Maekawa T, Sumikawa K. Inhalation of hydrogen gas protects against myocardial stunning and infarction in swine. Scand Cardiovasc J. 2012:46(3):183-9. doi:10.3109/14017431.2012.659676.

19. Yoshida A, Asanuma H, Sasaki H, Sanada S, Yamazaki S, Asano Y, et al. H(2) mediates cardioprotection via involvements of K(ATP) channels and permeability transition pores of mitochondria in dogs. Cardiovasc Drugs Ther. 2012;26(3):217-26. doi:10.1007/s10557-012-6381-5

20. Shinbo T, Kokubo K, Sato Y, Hagiri S, Hataishi R, Hirose M, et al. Breathing nitric oxide plus hydrogen gas reduces ischemia-reperfusion injury and nitrotyrosine production in murine heart. Am J Physiol Heart Circ Physiol. 2013;305(4):H542-50. doi:10.1152/ajpheart.00844.2012.

21. Sun Q, Kang Z, Cai J, Liu W, Liu Y, Zhang JH, et al. Hydrogen-rich saline protects myocardium against ischemia/reperfusion injury in rats. Exp Biol Med (Maywood). 2009;234(10):1212-9. doi:10.3181/0812-RM-349.

22. Zhang $Y$, Sun $Q$, He B, Xiao J, Wang Z, Sun X. Anti-inflammatory effect of hydrogen-rich saline in a rat model of regional myocardial ischemia and reperfusion. Int J Cardiol. 2011;148(1):91-5. doi:10.1016/ j.ijcard.2010.08.058.

23. Jing L, Wang Y, Zhao XM, Zhao B, Han JJ, Qin SC, et al. Cardioprotective Effect of Hydrogen-rich Saline on Isoproterenol-induced Myocardial Infarction in Rats. Heart Lung Circ. 2015;24(6):602-10. doi:10.1016/ j.hlc.2014.11.018

24. Zhang G, Gao S, Li X, Zhang L, Tan H, Xu L, et al. Pharmacological postconditioning with lactic acid and hydrogen rich saline alleviates myocardial reperfusion injury in rats. Sci Rep. 2015;5:9858. doi:10.1038/ srep09858.

25. Ohno K, Ito M, Ichihara M, Ito M. Molecular hydrogen as an emerging therapeutic medical gas for neurodegenerative and other diseases. Oxid Med Cell Longev. 2012;2012:353152. doi:10.1155/2012/353152.

26. Itoh T, Fujita Y, Ito M, Masuda A, Ohno K, Ichihara M, et al. Molecular hydrogen suppresses FcepsilonRI-mediated signal transduction and prevents degranulation of mast cells. Biochem Biophys Res Commun. 2009;389(4):651-6. doi:10.1016/j.bbrc.2009.09.047.

27. Itoh T, Hamada N, Terazawa R, Ito M, Ohno K, Ichihara M, et al. Molecular hydrogen inhibits lipopolysaccharide/interferon gamma-induced nitric oxide production through modulation of signal transduction in macrophages. Biochem Biophys Res Commun. 2011;411(1):143-9. doi:10.1016/ j.bbrc.2011.06.116.

28. Manaenko A, Lekic T, Ma Q, Zhang JH, Tang J. Hydrogen inhalation ameliorated mast cell-mediated brain injury after intracerebral hemorrhage in mice. Crit Care Med. 2013:41(5):1266-75. doi:10.1097/ CCM.0b013e31827711c9.

29. Chen Y, Jiang J, Miao H, Chen X, Sun X, Li Y. Hydrogen-rich saline attenuates vascular smooth muscle cell proliferation and neointimal hyperplasia by inhibiting reactive oxygen species production and inactivating the Ras-ERK1/2-MEK1/2 and Akt pathways. Int J Mol Med. 2013:31(3):597-606. doi:10.3892/ijmm.2013.1256.

30. Cardinal JS, Zhan J, Wang Y, Sugimoto R, Tsung A, McCurry KR, et al. Oral hydrogen water prevents chronic allograft nephropathy in rats. Kidney Int 2010;77(2):101-9. doi:10.1038/ki.2009.421.

31. Liu Q, Shen WF, Sun HY, Fan DF, Nakao A, Cai JM, et al. Hydrogen-rich saline protects against liver injury in rats with obstructive jaundice. Liver Int. 2010;30(7):958-68. doi:10.1111/j.1478-3231.2010.02254.x

32. Kasuyama K, Tomofuji T, Ekuni D, Tamaki N, Azuma T, Irie $K$, et al. Hydrogen-rich water attenuates experimental periodontitis in a rat model. J Clin Periodontol. 2011;38(12):1085-90. doi:10.1111/j.1600-051X.2011.01801.x.

33. Tanaka Y, Shigemura N, Kawamura T, Noda K, Isse K, Stolz DB, et al. Profiling molecular changes induced by hydrogen treatment of lung allografts prior to procurement. Biochem Biophys Res Commun. 2012;425(4):873-9. doi:10.1016/j.bbrc.2012.08.005.

34. Sun Y, Shuang F, Chen DM, Zhou RB. Treatment of hydrogen molecule abates oxidative stress and alleviates bone loss induced by modeled microgravity in rats. Osteoporos Int. 2013;24(3):969-78. doi:10.1007/ s00198-012-2028-4.

35. Xu XF, Zhang J. Saturated hydrogen saline attenuates endotoxin-induced acute liver dysfunction in rats. Physiol Res. 2013;62(4):395-403.

36. Li DZ, Zhang QX, Dong XX, Li HD, Ma X. Treatment with hydrogen molecules prevents RANKL-induced osteoclast differentiation associated with inhibition of ROS formation and inactivation of MAPK, AKT and NF-kappa B pathways in murine RAW264.7 cells. J Bone Miner Metab. 2014:32(5):494-504. doi:10.1007/s00774-013-0530-1.

37. Guo SX, Fang Q, You CG, Jin YY, Wang XG, Hu XL, et al. Effects of hydrogen-rich saline on early acute kidney injury in severely burned rats by suppressing oxidative stress induced apoptosis and inflammation. J Transl Med. 2015:13:183. doi:10.1186/s12967-015-0548-3.

38. Liu X, Chen Z, Mao N, Xie Y. The protective of hydrogen on stress-induced gastric ulceration. Int Immunopharmacol. 2012;13(2):197-203. doi:10.1016/ j.intimp.2012.04.004.

39. Chen Q, Chen P, Zhou S, Yan X, Zhang J, Sun X, et al. Hydrogen-rich saline attenuated neuropathic pain by reducing oxidative stress. Can J Neurol Sci. 2013;40(6):857-63.

40. Wu F, Qiu Y, Ye G, Luo H, Jiang J, Yu F, et al. Treatment with hydrogen molecule attenuates cardiac dysfunction in streptozotocin-induced diabetic mice. Cardiovasc Pathol. 2015:24:294-303. doi:10.1016/j.carpath.2015.04.008.

41. Zhai $Y$, Zhou X, Dai Q, Fan Y, Huang X. Hydrogen-rich saline ameliorates lung injury associated with cecal ligation and puncture-induced sepsis in rats. Exp Mol Pathol. 2015;98(2):268-76. doi:10.1016/j.yexmp.2015.03.005.

42. Sun $H$, Chen L, Zhou W, Hu L, Li L, Tu Q, et al. The protective role of hydrogen-rich saline in experimental liver injury in mice. J Hepatol. 2011;54(3):471-80. doi:10.1016/j.jhep.2010.08.011.

43. Wang C, Li J, Liu Q, Yang R, Zhang JH, Cao YP, et al. Hydrogen-rich saline reduces oxidative stress and inflammation by inhibit of JNK and NF-kappaB activation in a rat model of amyloid-beta-induced Alzheimer's disease. Neurosci Lett. 2011;491(2):127-32. doi:10.1016/j.neulet.2011.01.022.

44. Iio A, Ito M, Itoh T, Terazawa R, Fujita Y, Nozawa Y, et al. Molecular hydrogen attenuates fatty acid uptake and lipid accumulation through downregulating CD36 expression in HepG2 cells. Med Gas Res. 2013;3(1):6. doi:10.1186/2045-9912-3-6.

45. Shin MH, Park R, Nojima H, Kim HC, Kim YK, Chung JH. Atomic hydrogen surrounded by water molecules, $\mathrm{H}(\mathrm{H} 2 \mathrm{O}) \mathrm{m}$, modulates basal and UV-induced gene expressions in human skin in vivo. PLoS One. 2013;8(4):e61696. doi:10.1371/journal.pone.0061696.

46. Liu YQ, Liu YF, Ma XM, Xiao YD, Wang YB, Zhang MZ, et al. Hydrogen-rich saline attenuates skin ischemia/reperfusion induced apoptosis via regulating Bax/BCl-2 ratio and ASK-1/JNK pathway. J Plast Reconstr Aesthet Surg. 2015;68(7):e147-56. doi:10.1016/j.bjps.2015.03.001. 
47. Zhang JY, Song SD, Pang Q, Zhang RY, Wan Y, Yuan DW, et al. Hydrogen-rich water protects against acetaminophen-induced hepatotoxicity in mice. World J Gastroenterol. 2015;21(14):4195-209. doi:10.3748/wjg.v21.i14.4195.

48. Hong Y, Shao A, Wang J, Chen S, Wu H, McBride DW, et al. Neuroprotective effect of hydrogen-rich saline against neurologic damage and apoptosis in early brain injury following subarachnoid hemorrhage: possible role of the Akt/GSK3beta signaling pathway. PLoS One. 2014;9(4):e96212. doi:10.1371/ journal.pone.0096212.

49. Guo SX, Jin YY, Fang Q, You CG, Wang XG, Hu XL, et al. Beneficial effects of hydrogen-rich saline on early burn-wound progression in rats. PLoS One. 2015;10(4):e0124897. doi:10.1371/journal.pone.0124897.

50. Wei L, Ge L, Qin S, Shi Y, Du C, Du H, et al. Hydrogen-rich saline protects retina against glutamate-induced excitotoxic injury in guinea pig. Exp Eye Res. 2012:94(1):117-27. doi:10.1016/j.exer.2011.11.016.

51. Abe T, Li XK, Yazawa K, Hatayama N, Xie L, Sato B, et al. Hydrogen-rich University of Wisconsin solution attenuates renal cold ischemia-reperfusion injury. Transplantation. 2012;94(1):14-21. doi:10.1097/TP.0b013e318255f8be.

52. Noda K, Shigemura N, Tanaka Y, Kawamura T, Hyun Lim S, Kokubo K, et al. A novel method of preserving cardiac grafts using a hydrogen-rich water bath. J Heart Lung Transplant. 2013;32(2):241-50. doi:10.1016/j.healun.2012.11.004.

53. Huang CS, Kawamura T, Peng X, Tochigi N, Shigemura N, Billiar TR, et al. Hydrogen inhalation reduced epithelial apoptosis in ventilator-induced lung injury via a mechanism involving nuclear factor-kappa B activation. Biochem Biophys Res Commun. 2011;408(2):253-8. doi:10.1016/j.bbrc.2011.04.008.

54. Song G, Tian H, Liu J, Zhang H, Sun X, Qin S. H2 inhibits TNF-alpha-induced lectin-like oxidized LDL receptor-1 expression by inhibiting nuclear factor kappaB activation in endothelial cells. Biotechnol Lett. 2011;33(9):1715-22. doi:10.1007/s10529-011-0630-8

55. Kubota M, Shimmura S, Kubota S, Miyashita H, Kato N, Noda K, et al. Hydrogen and $\mathrm{N}$-acetyl-L-cysteine rescue oxidative stress-induced angiogenesis in a mouse corneal alkali-burn model. Invest Ophthalmol Vis Sci. 2011:52(1):427-33. doi:10.1167/iovs.10-6167.

56. Ji Q, Hui K, Zhang L, Sun X, Li W, Duan M. The effect of hydrogen-rich saline on the brain of rats with transient ischemia. J Surg Res. 2011;168(1):e95-101. doi:10.1016/j.jss.2011.01.057.

57. Shen L, Wang J, Liu K, Wang C, Wang C, Wu H, et al. Hydrogen-rich saline is cerebroprotective in a rat model of deep hypothermic circulatory arrest. Neurochem Res. 2011;36(8):1501-11. doi:10.1007/s11064-011-0476-4.

58. Qin ZX, Yu P, Qian DH, Song MB, Tan H, Yu Y, et al. Hydrogen-rich saline prevents neointima formation after carotid balloon injury by suppressing ROS and the TNF-alpha/NF-kappaB pathway. Atherosclerosis. 2012;220(2):343-50. doi:10.1016/j.atherosclerosis.2011.11.002.

59. Song G, Tian H, Qin S, Sun X, Yao S, Zong C, et al. Hydrogen decreases athero-susceptibility in apolipoprotein B-containing lipoproteins and aorta of apolipoprotein E knockout mice. Atherosclerosis. 2012;221(1):55-65. doi:10.1016/j.atherosclerosis.2011.11.043.

60. Zheng $H$, Yu YS. Chronic hydrogen-rich saline treatment attenuates vascular dysfunction in spontaneous hypertensive rats. Biochem Pharmacol. 2012;83(9):1269-77. doi:10.1016/j.bcp.2012.01.031.

61. Hong Y, Guo S, Chen S, Sun C, Zhang J, Sun X. Beneficial effect of hydrogen-rich saline on cerebral vasospasm after experimental subarachnoid hemorrhage in rats. J Neurosci Res. 2012;90(8):1670-80. doi:10.1002/jnr.22739.

62. Guo JD, Li L, Shi YM, Wang HD, Hou SX. Hydrogen water consumption prevents osteopenia in ovariectomized rats. Br J Pharmacol. 2013;168(6):1412-20. doi:10.1111/bph.12036.

63. Xiao M, Zhu T, Wang T, Wen FQ. Hydrogen-rich saline reduces airway remodeling via inactivation of NF-kappaB in a murine model of asthma. Eur Rev Med Pharmacol Sci. 2013;17(8):1033-43.

64. Liu GD, Zhang H, Wang L, Han Q, Zhou SF, Liu P. Molecular hydrogen regulates the expression of miR-9, miR-21 and miR-199 in LPS-activated retinal microglia cells. Int J Ophthalmol. 2013;6(3):280-5. doi:10.3980/ j.issn.2222-3959.2013.03.05.

65. Li FY, Zhu SX, Wang ZP, Wang H, Zhao Y, Chen GP. Consumption of hydrogen-rich water protects against ferric nitrilotriacetate-induced nephrotoxicity and early tumor promotional events in rats. Food Chem Toxicol. 2013;61:248-54. doi:10.1016/j.fct.2013.10.004.

66. Zhuang Z, Sun XJ, Zhang X, Liu HD, You WC, Ma CY, et al. Nuclear factorkappaB/BCl-XL pathway is involved in the protective effect of hydrogen-rich saline on the brain following experimental subarachnoid hemorrhage in rabbits. J Neurosci Res. 2013;91(12):1599-608. doi:10.1002/jnr.23281.
67. Tan YC, Xie F, Zhang HL, Zhu YL, Chen K, Tan HM, et al. Hydrogen-rich saline attenuates postoperative liver failure after major hepatectomy in rats. Clin Res Hepatol Gastroenterol. 2014;38(3):337-45. doi:10.1016/ j.clinre.2013.11.007.

68. Zhang J, Wu Q, Song S, Wan Y, Zhang R, Tai M, et al. Effect of hydrogenrich water on acute peritonitis of rat models. Int Immunopharmacol. 2014;21(1):94-101. doi:10.1016/j.intimp.2014.04.011.

69. Xin HG, Zhang BB, Wu ZQ, Hang XF, Xu WS, Ni W, et al. Consumption of hydrogen-rich water alleviates renal injury in spontaneous hypertensive rats. Mol Cell Biochem. 2014;392(1-2):117-24. doi:10.1007/s11010-014-2024-4.

70. Wang X, Yu P, Yong Y, Liu X, Jiang J, Liu D, et al. Hydrogen-rich saline resuscitation alleviates inflammation induced by severe burn with delayed resuscitation. Burns. 2015;41(2):379-85. doi:10.1016/j.burns.2014.07.012.

71. Zhang CB, Tang YC, Xu XJ, Guo SX, Wang HZ. Hydrogen gas inhalation protects against liver ischemia/reperfusion injury by activating the NF-kappaB signaling pathway. Exp Ther Med. 2015;9(6):2114-20. doi:10.3892/etm.2015.2385

72. Shi Q, Liao KS, Zhao KL, Wang WX, Zuo T, Deng WH, et al. Hydrogen-rich saline attenuates acute renal injury in sodium taurocholate-induced severe acute pancreatitis by inhibiting ROS and NF-kappaB pathway. Mediators Inflamm. 2015:2015:685043. doi:10.1155/2015/685043.

73. Shao A, Wu H, Hong Y, Tu S, Sun X, Wu Q et al. Hydrogen-Rich Saline Attenuated Subarachnoid Hemorrhage-Induced Early Brain Injury in Rats by Suppressing Inflammatory Response: Possible Involvement of NF-kappaB Pathway and NLRP3 Inflammasome. Mol Neurobiol. 2015. doi:10.1007/s12035-015-9242-y.

74. Chen X, Liu Q, Wang D, Feng S, Zhao Y, Shi Y, et al. Protective Effects of Hydrogen-Rich Saline on Rats with Smoke Inhalation Injury. Oxid Med Cell Longev. 2015;2015:106836. doi:10.1155/2015/106836.

75. Kohama K, Yamashita H, Aoyama-Ishikawa M, Takahashi T, Billiar TR, Nishimura T, et al. Hydrogen inhalation protects against acute lung injury induced by hemorrhagic shock and resuscitation. Surgery. 2015;158(2):399_ 407. doi:10.1016/j.surg.2015.03.038.

76. Ren JD, Ma J, Hou J, Xiao WJ, Jin WH, Wu J, et al. Hydrogen-rich saline inhibits NLRP3 inflammasome activation and attenuates experimental acute pancreatitis in mice. Mediators Inflamm. 2014;2014:930894. doi:10.1155/2014/930894.

77. Liu FT, Xu SM, Xiang ZH, Li XN, Li J, Yuan HB, et al. Molecular hydrogen suppresses reactive astrogliosis related to oxidative injury during spinal cord injury in rats. CNS Neurosci Ther. 2014;20(8):778-86. doi:10.1111/cns.12258.

78. Kishimoto Y, Kato T, Ito M, Azuma Y, Fukasawa Y, Ohno K, et al. Hydrogen ameliorates pulmonary hypertension in rats by anti-inflammatory and antioxidant effects. J Thorac Cardiovasc Surg. 2015;150:645-54. doi:10.1016/ j.jtcvs.2015.05.052

79. Zhang L, Shu R, Wang C, Wang H, Li N, Wang G. Hydrogen-rich saline controls remifentanil-induced hypernociception and NMDA receptor NR1 subunit membrane trafficking through GSK-3beta in the DRG in rats. Brain Res Bull. 2014;106:47-55. doi:10.1016/j.brainresbull.2014.05.005.

80. Xie K, Wang W, Chen H, Han H, Liu D, Wang G, et al. Hydrogen-Rich Medium Attenuated Lipopolysaccharide-Induced Monocyte-Endothelial Cell Adhesion and Vascular Endothelial Permeability via Rho-Associated Coiled-Coil Protein Kinase. Shock. 2015:44(1):58-64. doi:10.1097/SHK.0000000000000365.

81. Spulber S, Edoff K, Hong L, Morisawa S, Shirahata S, Ceccatelli S. Molecular hydrogen reduces LPS-induced neuroinflammation and promotes recovery from sickness behaviour in mice. PLoS One. 2012;7(7):e42078. doi:10.1371/ journal.pone.0042078.

82. Kawamura T, Wakabayashi N, Shigemura N, Huang CS, Masutani K, Tanaka Y, et al. Hydrogen gas reduces hyperoxic lung injury via the Nrf2 pathway in vivo. Am J Physiol Lung Cell Mol Physiol. 2013;304(10):L646-56. doi:10.1152/ ajplung.00164.2012.

83. Zhai X, Chen X, Shi J, Shi D, Ye Z, Liu W, et al. Lactulose ameliorates cerebral ischemia-reperfusion injury in rats by inducing hydrogen by activating Nrf2 expression. Free Radic Biol Med. 2013;65:731-41. doi:10.1016/ j.freeradbiomed.2013.08.004.

84. Xie Q, Li XX, Zhang $P$, Li JC, Cheng Y, Feng $Y L$, et al. Hydrogen gas protects against serum and glucose deprivationinduced myocardial injury in $\mathrm{H} 9 \mathrm{c} 2$ cells through activation of the NFE2related factor 2/heme oxygenase 1 signaling pathway. Mol Med Rep. 2014;10(2):1143-9. doi:10.3892/mmr.2014.2283.

85. Song G, Zong C, Zhang Z, Yu Y, Yao S, Jiao P, et al. Molecular Hydrogen stabilizes atherosclerotic plaque in low-density lipoprotein receptor knockout mice. Free Radic Biol Med. 2015;87:58-68. doi:10.1016/ j.freeradbiomed.2015.06.018. 
86. Li Y, Xie K, Chen H, Wang G, Yu Y. Hydrogen gas inhibits high-mobility group box 1 release in septic mice by upregulation of heme oxygenase 1. J Surg Res. 2015;196(1):136-48. doi:10.1016/j.jss.2015.02.042.

87. Li Y, Li Q, Chen H, Wang T, Liu L, Wang G, et al. Hydrogen Gas Alleviates the Intestinal Injury Caused by Severe Sepsis in Mice by Increasing the Expression of Heme Oxygenase-1. Shock. 2015;44(1):90-8. doi:10.1097/SHK.0000000000000382.

88. Kawamura T, Huang CS, Peng X, Masutani K, Shigemura N, Billiar TR, et al. The effect of donor treatment with hydrogen on lung allograft function in rats. Surgery. 2011;150(2):240-9. doi:10.1016/j.surg.2011.05.019.

89. Buchholz BM, Masutani K, Kawamura T, Peng X, Toyoda Y, Billiar TR, et al. Hydrogen-enriched preservation protects the isogeneic intestinal graft and amends recipient gastric function during transplantation. Transplantation. 2011:92(9):985-92. doi:10.1097/TP.0b013e318230159d.

90. Chen HG, Xie KL, Han HZ, Wang WN, Liu DQ, Wang GL, et al. Heme oxygenase-1 mediates the anti-inflammatory effect of molecular hydrogen in LPS-stimulated RAW 264.7 macrophages. Int J Surg. 2013;11(10):1060-6. doi:10.1016/j.jijsu.2013.10.007.

91. Lin Y, Zhang W, Qi F, Cui W, Xie Y, Shen W. Hydrogen-rich water regulates cucumber adventitious root development in a heme oxygenase-1/carbon monoxide-dependent manner. J Plant Physiol. 2014;171(2):1-8. doi:10.1016/j.jplph.2013.08.009.

92. Chen Y, Chen H, Xie K, Liu L, Li Y, Yu Y, et al. H Treatment Attenuated Pain Behavior and Cytokine Release Through the HO-1/CO Pathway in a Rat Model of Neuropathic Pain. Inflammation. 2015;38:1835-46. doi:10.1007/s10753-015-0161-X.

93. Yu J, Zhang W, Zhang R, Ruan X, Ren P, Lu B. Lactulose accelerates liver regeneration in rats by inducing hydrogen. J Surg Res. 2015;195(1):128-35. doi:10.1016/j.jss.2015.01.034.

94. Wei R, Zhang R, Xie Y, Shen L, Chen F. Hydrogen Suppresses Hypoxia/ Reoxygenation-Induced Cell Death in Hippocampal Neurons Through Reducing Oxidative Stress. Cell Physiol Biochem. 2015;36(2):585-98. doi:10.1159/000430122.

95. Matsumoto A, Yamafuji M, Tachibana T, Nakabeppu Y, Noda M, Nakaya H. Oral 'hydrogen water' induces neuroprotective ghrelin secretion in mice. Sci Rep. 2013;3:3273. doi:10.1038/srep03273

96. Yoritaka A, Takanashi M, Hirayama M, Nakahara T, Ohta S, Hattori N. Pilot study of $\mathrm{H}(2)$ therapy in Parkinson's disease: a randomized double-blind placebo-controlled trial. Mov Disord. 2013;28(6):836-9. doi:10.1002/mds.25375.

97. Fujita K, Seike T, Yutsudo N, Ohno M, Yamada H, Yamaguchi $H$, et al. Hydrogen in drinking water reduces dopaminergic neuronal loss in the 1-methyl-4-phenyl-1,2,3,6-tetrahydropyridine mouse model of Parkinson's disease. PLoS One. 2009;4(9):e7247. doi:10.1371/journal.pone.0007247.

98. Fukuda K, Asoh S, Ishikawa M, Yamamoto Y, Ohsawa I, Ohta S. Inhalation of hydrogen gas suppresses hepatic injury caused by ischemia/reperfusion through reducing oxidative stress. Biochem Biophys Res Commun. 2007;361(3):670-4. doi:10.1016/j.bbrc.2007.07.088

99. Chen $\mathrm{CH}$, Manaenko A, Zhan Y, Liu WW, Ostrowki RP, Tang J, et al, Hydrogen gas reduced acute hyperglycemia-enhanced hemorrhagic transformation in a focal ischemia rat model. Neuroscience. 2010;169(1):402-14. doi:10.1016/j.neuroscience.2010.04.043.

100. Hugyecz M, Mracsko E, Hertelendy P, Farkas E, Domoki F, Bari F. Hydrogen supplemented air inhalation reduces changes of prooxidant enzyme and gap junction protein levels after transient global cerebral ischemia in the rat hippocampus. Brain Res. 2011;1404:31-8. doi:10.1016/j.brainres.2011.05.068.

101. Ono H, Nishijima Y, Adachi N, Tachibana S, Chitoku S, Mukaihara S, et al. Improved brain MRI indices in the acute brain stem infarct sites treated with hydroxyl radical scavengers, Edaravone and hydrogen, as compared to Edaravone alone. A non-controlled study. Med Gas Res. 2011;1(1):12. doi:10.1186/2045-9912-1-12.

102. Liu Y, Liu W, Sun X, Li R, Sun Q, Cai J, et al. Hydrogen saline offers neuroprotection by reducing oxidative stress in a focal cerebral ischemiareperfusion rat model. Med Gas Res. 2011;1(1):15. doi:10.1186/2045-9912-1-15.

103. Li J, Dong Y, Chen H, Han H, Yu Y, Wang G, et al. Protective effects of hydrogen-rich saline in a rat model of permanent focal cerebral ischemia via reducing oxidative stress and inflammatory cytokines. Brain Res. 2012;1486:103-11. doi:10.1016/j.brainres.2012.09.031.

104. Nagatani K, Wada K, Takeuchi S, Kobayashi H, Uozumi Y, Otani N, et al. Effect of hydrogen gas on the survival rate of mice following global cerebral ischemia. Shock. 2012:37(6):645-52. doi:10.1097/SHK.0b013e31824ed57c.
105. Ge P, Zhao J, Li S, Ding Y, Yang F, Luo Y. Inhalation of hydrogen gas attenuates cognitive impairment in transient cerebral ischemia via inhibition of oxidative stress. Neurol Res. 2012;34(2):187-94. doi:10.1179/ $1743132812 Y .0000000002$.

106. Nagatani K, Nawashiro H, Takeuchi S, Tomura S, Otani N, Osada H, et al. Safety of intravenous administration of hydrogen-enriched fluid in patients with acute cerebral ischemia: initial clinical studies. Med Gas Res. 2013;3(1):13. doi:10.1186/2045-9912-3-13

107. Olah O, Toth-Szuki V, Temesvari P, Bari F, Domoki F. Delayed neurovascular dysfunction is alleviated by hydrogen in asphyxiated newborn pigs. Neonatology. 2013;104(2):79-86. doi:10.1159/000348445.

108. Cui $Y$, Zhang $H$, Ji M, Jia M, Chen $H$, Yang J, et al. Hydrogen-rich saline attenuates neuronal ischemia-reperfusion injury by protecting mitochondrial function in rats. J Surg Res. 2014;192(2):564-72. doi:10.1016/j.jss.2014.05.060

109. Han L, Tian R, Yan H, Pei L, Hou Z, Hao S, et al. Hydrogen-rich water protects against ischemic brain injury in rats by regulating calcium buffering proteins. Brain Res. 2015;1615:129-38. doi:10.1016/j.brainres.2015.04.038.

110. Takeuchi S, Nagatani K, Otani N, Nawashiro H, Sugawara T, Wada K, et al. Hydrogen improves neurological function through attenuation of blood-brain barrier disruption in spontaneously hypertensive stroke-prone rats. BMC Neurosci. 2015;16(1):22. doi:10.1186/s12868-015-0165-3.

111. Zhuang Z, Zhou ML, You WC, Zhu L, Ma CY, Sun XJ, et al. Hydrogen-rich saline alleviates early brain injury via reducing oxidative stress and brain edema following experimental subarachnoid hemorrhage in rabbits. BMC Neurosci. 2012;13:47. doi:10.1186/1471-2202-13-47.

112. Zhan Y, Chen C, Suzuki H, Hu Q, Zhi X, Zhang JH. Hydrogen gas ameliorates oxidative stress in early brain injury after subarachnoid hemorrhage in rats. Crit Care Med. 2012:40(4):1291-6. doi:10.1097/CCM.0b013e31823da96d.

113. Takeuchi S, Mori K, Arimoto H, Fujii K, Nagatani K, Tomura S, et al. Effects of intravenous infusion of hydrogen-rich fluid combined with intra-cisternal infusion of magnesium sulfate in severe aneurysmal subarachnoid hemorrhage: study protocol for a randomized controlled trial. BMC Neurol. 2014;14:176. doi:10.1186/s12883-014-0176-1.

114. Ji X, Liu W, Xie K, Liu W, Qu Y, Chao X, et al. Beneficial effects of hydrogen gas in a rat model of traumatic brain injury via reducing oxidative stress. Brain Res. 2010;1354:196-205. doi:10.1016/j.brainres.2010.07.038.

115. Eckermann JM, Chen W, Jadhav V, Hsu FP, Colohan AR, Tang J, et al. Hydrogen is neuroprotective against surgically induced brain injury. Med Gas Res. 2011;1(1):7. doi:10.1186/2045-9912-1-7.

116. Hou Z, Luo W, Sun X, Hao S, Zhang Y, Xu F, et al. Hydrogen-rich saline protects against oxidative damage and cognitive deficits after mild traumatic brain injury. Brain Res Bull. 2012;88(6):560-5. doi:10.1016/ j.brainresbull.2012.06.006

117. Ji X, Tian Y, Xie K, Liu W, Qu Y, Fei Z. Protective effects of hydrogen-rich saline in a rat model of traumatic brain injury via reducing oxidative stress. J Surg Res. 2012;178(1):e9-16. doi:10.1016/j.jss.2011.12.038.

118. Dohi K, Kraemer BC, Erickson MA, McMillan PJ, Kovac A, Flachbartova Z, et al. Molecular hydrogen in drinking water protects against neurodegenerative changes induced by traumatic brain injury. PLoS One. 2014;9(9):e108034. doi:10.1371/journal.pone.0108034.

119. Fu Y, Ito M, Fujita Y, Ito M, Ichihara M, Masuda A, et al. Molecular hydrogen is protective against 6-hydroxydopamine-induced nigrostriatal degeneration in a rat model of Parkinson's disease. Neurosci Lett. 2009;453(2):81-5. doi:10.1016/j.neulet.2009.02.016

120. Li J, Wang C, Zhang JH, Cai JM, Cao YP, Sun XJ. Hydrogen-rich saline improves memory function in a rat model of amyloid-beta-induced Alzheimer's disease by reduction of oxidative stress. Brain Res. 2010;1328:152-61. doi:10.1016/j.brainres.2010.02.046.

121. Nagata K, Nakashima-Kamimura N, Mikami T, Ohsawa I, Ohta S. Consumption of molecular hydrogen prevents the stress-induced impairments in hippocampus-dependent learning tasks during chronic physical restraint in mice. Neuropsychopharmacology. 2009;34(2):501-8. doi:10.1038/npp.2008.95.

122. Gu Y, Huang CS, Inoue T, Yamashita T, Ishida T, Kang KM, et al. Drinking hydrogen water ameliorated cognitive impairment in senescence-accelerated mice. J Clin Biochem Nutr. 2010;46(3):269-76. doi:10.3164/jcbn.10-19.

123. Liu L, Xie K, Chen H, Dong X, Li Y, Yu Y, et al. Inhalation of hydrogen gas attenuates brain injury in mice with cecal ligation and puncture via inhibiting neuroinflammation, oxidative stress and neuronal apoptosis. Brain Res. 2014;1589:78-92. doi:10.1016/j.brainres.2014.09.030. 
124. Ueda Y, Nakajima A, Oikawa T. Hydrogen-related enhancement of in vivo antioxidant ability in the brain of rats fed coral calcium hydride. Neurochem Res. 2010;35(10):1510-5. doi:10.1007/s11064-010-0204-5

125. Kashiwagi T, Yan H, Hamasaki T, Kinjo T, Nakamichi N, Teruya K, et al. Electrochemically reduced water protects neural cells from oxidative damage. Oxid Med Cell Longev. 2014;2014:869121. doi:10.1155/2014/869121.

126. Huang Y, Xie K, Li J, Xu N, Gong G, Wang G, et al. Beneficial effects of hydrogen gas against spinal cord ischemia-reperfusion injury in rabbits. Brain Res. 2011;1378:125-36. doi:10.1016/j.brainres.2010.12.071.

127. Zhou L, Wang X, Xue W, Xie K, Huang Y, Chen H, et al. Beneficial effects of hydrogen-rich saline against spinal cord ischemia-reperfusion injury in rabbits. Brain Res. 2013;1517:150-60. doi:10.1016/j.brainres.2013.04.007.

128. Chen C, Chen Q, Mao Y, Xu S, Xia C, Shi X, et al. Hydrogen-rich saline protects against spinal cord injury in rats. Neurochem Res. 2010;35(7):1111-8. doi:10.1007/s11064-010-0162-y.

129. Ge Y, Wu F, Sun X, Xiang Z, Yang L, Huang S, et al. Intrathecal infusion of hydrogen-rich normal saline attenuates neuropathic pain via inhibition of activation of spinal astrocytes and microglia in rats. PLoS One. 2014;9(5):e97436. doi:10.1371/journal.pone.0097436.

130. Kawaguchi M, Satoh Y, Otsubo Y, Kazama T. Molecular hydrogen attenuates neuropathic pain in mice. PLoS One. 2014;9(6):e100352. doi:10.1371/ journal.pone.0100352.

131. Zhang L, Shu R, Wang H, Yu Y, Wang C, Yang M, et al. Hydrogen-rich saline prevents remifentanil-induced hyperalgesia and inhibits MnSOD nitration via regulation of NR2B-containing NMDA receptor in rats. Neuroscience. 2014;280:171-80. doi:10.1016/j.neuroscience.2014.09.024.

132. Shu RC, Zhang LL, Wang CY, Li N, Wang HY, Xie KL, et al. Spinal peroxynitrite contributes to remifentanil-induced postoperative hyperalgesia via enhancement of divalent metal transporter 1 without iron-responsive element-mediated iron accumulation in rats. Anesthesiology. 2015;122(4):908-20. doi:10.1097/ALN.0000000000000562.

133. Oharazawa H, Igarashi T, Yokota T, Fujii H, Suzuki H, Machide M, et al. Protection of the retina by rapid diffusion of hydrogen: administration of hydrogen-loaded eye drops in retinal ischemia-reperfusion injury. Invest Ophthalmol Vis Sci. 2010;51(1):487-92. doi:10.1167/iovs.09-4089.

134. Liu H, Hua N, Xie K, Zhao T, Yu Y. Hydrogen-rich saline reduces cell death through inhibition of DNA oxidative stress and overactivation of poly (ADPribose) polymerase-1 in retinal ischemia-reperfusion injury. Mol Med Rep. 2015;12(2):2495-502. doi:10.3892/mmr.2015.3731.

135. Xiao X, Cai J, Xu J, Wang R, Cai J, Liu Y, et al. Protective effects of hydrogen saline on diabetic retinopathy in a streptozotocin-induced diabetic rat model. J Ocul Pharmacol Ther. 2012;28(1):76-82. doi:10.1089/jop.2010.0129.

136. Feng $Y$, Wang $R$, Xu J, Sun J, Xu T, Gu Q, et al. Hydrogen-rich saline prevents early neurovascular dysfunction resulting from inhibition of oxidative stress in STZ-diabetic rats. Curr Eye Res. 2013;38(3):396-404 doi:10.3109/02713683.2012.748919.

137. Huang L, Zhao S, Zhang JH, Sun X. Hydrogen saline treatment attenuates hyperoxia-induced retinopathy by inhibition of oxidative stress and reduction of VEGF expression. Ophthalmic Res. 2012;47(3):122-7. doi:10.1159/000329600

138. Feng $M$, Wang $X H$, Yang $X B$, Xiao $Q$, Jiang FG. Protective effect of saturated hydrogen saline against blue light-induced retinal damage in rats. Int J Ophthalmol. 2012;5(2):151-7. doi:10.3980/j.issn.2222-3959.2012.02.07.

139. Tian L, Zhang L, Xia F, An J, Sugita Y, Zhang Z. Hydrogen-rich saline ameliorates the retina against light-induced damage in rats. Med Gas Res. 2013:3(1):19. doi:10.1186/2045-9912-3-19.

140. Yokota T, Kamimura N, Igarashi T, Takahashi H, Ohta S, Oharazawa H. Protective effect of molecular hydrogen against oxidative stress caused by peroxynitrite derived from nitric oxide in rat retina. Clin Experiment Ophthalmol. 2015:43:568-77. doi:10.1111/ceo.12525.

141. Sun JC, Xu T, Zuo Q, Wang RB, Qi AQ, Cao WL, et al. Hydrogen-rich saline promotes survival of retinal ganglion cells in a rat model of optic nerve crush. PLoS One. 2014;9(6):e99299. doi:10.1371/journal.pone.0099299.

142. Yang CX, Yan H, Ding TB. Hydrogen saline prevents selenite-induced cataract in rats. Mol Vis. 2013;19:1684-93.

143. Kikkawa YS, Nakagawa T, Horie RT, Ito J. Hydrogen protects auditory hair cells from free radicals. Neuroreport. 2009;20(7):689-94. doi:10.1097/ WNR.0b013e32832a5c68

144. Taura A, Kikkawa YS, Nakagawa T, Ito J. Hydrogen protects vestibular hair cells from free radicals. Acta Otolaryngol Suppl. 2010;130(563):95-100. doi:10.3109/00016489.2010.486799.
145. Lin Y, Kashio A, Sakamoto T, Suzukawa K, Kakigi A, Yamasoba T. Hydrogen in drinking water attenuates noise-induced hearing loss in guinea pigs. Neurosci Lett. 2011;487(1):12-6. doi:10.1016/j.neulet.2010.09.064.

146. Zhou Y, Zheng H, Ruan F, Chen X, Zheng G, Kang M, et al. Hydrogen-rich saline alleviates experimental noise-induced hearing loss in guinea pigs. Neuroscience. 2012;209:47-53. doi:10.1016/j.neuroscience.2012.02.028.

147. Chen L, Yu N, Lu Y, Wu L, Chen D, Guo W, et al. Hydrogen-saturated saline protects intensive narrow band noise-induced hearing loss in guinea pigs through an antioxidant effect. PLoS One. 2014;9(6):e100774. doi:10.1371/journal.pone.0100774.

148. Kurioka T, Matsunobu T, Satoh Y, Niwa K, Shiotani A. Inhaled hydrogen gas therapy for prevention of noise-induced hearing loss through reducing reactive oxygen species. Neurosci Res. 2014;89:69-74. doi:10.1016/j.neures.2014.08.009.

149. Qu J, Li X, Wang J, Mi W, Xie K, Qiu J. Inhalation of hydrogen gas attenuates cisplatin-induced ototoxicity via reducing oxidative stress. Int J Pediatr Otorhinolaryngol. 2012;76(1):111-5. doi:10.1016/j.ijporl.2011.10.014.

150. Kikkawa YS, Nakagawa T, Taniguchi M, Ito J. Hydrogen protects auditory hair cells from cisplatin-induced free radicals. Neurosci Lett. 2014:579:125-9. doi:10.1016/j.neulet.2014.07.025.

151. Qu J, Gan YN, Xie KL, Liu WB, Wang YF, Hei RY, et al. Inhalation of hydrogen gas attenuates ouabain-induced auditory neuropathy in gerbils. Acta Pharmacol Sin. 2012;33(4):445-51. doi:10.1038/aps.2011.190.

152. Tomofuji T, Kawabata $Y$, Kasuyama K, Endo $Y$, Yoneda $T$, Yamane $M$, et al. Effects of hydrogen-rich water on aging periodontal tissues in rats. Sci Rep. 2014;4:5534. doi:10.1038/srep05534.

153. Shi J, Yao F, Zhong C, Pan X, Yang Y, Lin Q. Hydrogen saline is protective for acute lung ischaemia/reperfusion injuries in rats. Heart Lung Circ. 2012;21(9):556-63. doi:10.1016/j.hlc.2012.05.782.

154. Li H, Zhou R, Liu J, Li Q, Zhang J, Mu J, et al. Hydrogen-rich saline attenuates lung ischemia-reperfusion injury in rabbits. J Surg Res. 2012;174(1):e11-6. doi:10.1016/j.jss.2011.10.001.

155. Zheng J, Liu K, Kang Z, Cai J, Liu W, Xu W, et al. Saturated hydrogen saline protects the lung against oxygen toxicity. Undersea Hyperb Med. 2010;37(3):185-92.

156. Sun Q, Cai J, Liu S, Liu Y, Xu W, Tao H, et al. Hydrogen-rich saline provides protection against hyperoxic lung injury. J Surg Res. 2011;165(1):e43-9. doi:10.1016/j.jss.2010.09.024

157. Huang CS, Kawamura T, Lee S, Tochigi N, Shigemura N, Buchholz BM, et al. Hydrogen inhalation ameliorates ventilator-induced lung injury. Crit Care. 2010;14(6):R234. doi:10.1186/cc9389.

158. Liu $H$, Liang $X$, Wang $D$, Zhang $H$, Liu L, Chen $H$, et al. Combination therapy with nitric oxide and molecular hydrogen in a murine model of acute lung injury. Shock. 2015;43(5):504-11. doi:10.1097/SHK.0000000000000316.

159. Mao YF, Zheng XF, Cai JM, You XM, Deng XM, Zhang JH, et al. Hydrogen-rich saline reduces lung injury induced by intestinal ischemia/reperfusion in rats. Biochem Biophys Res Commun. 2009;381(4):602-5. doi:10.1016/j.bbrc.2009.02.105.

160. Fang Y, Fu XJ, Gu C, Xu P, Wang Y, Yu WR, et al. Hydrogen-rich saline protects against acute lung injury induced by extensive burn in rat model. J Burn Care Res. 2011;32(3):e82-91. doi:10.1097/BCR.0b013e318217f84f.

161. Liu S, Liu K, Sun Q, Liu W, Xu W, Denoble P, et al. Consumption of hydrogen water reduces paraquat-induced acute lung injury in rats. J Biomed Biotechnol. 2011:2011:305086. doi:10.1155/2011/305086.

162. Sato C, Kamijo Y, Yoshimura K, Nagaki T, Yamaya T, Asakuma S, et al. Effects of hydrogen water on paraquat-induced pulmonary fibrosis in mice. Kitasato Med J. 2015;45(1):9-16.

163. Ning $Y$, Shang $Y$, Huang $H$, Zhang J, Dong $Y, X u$ W, et al. Attenuation of cigarette smoke-induced airway mucus production by hydrogen-rich saline in rats. PLoS One. 2013;8(12):e83429. doi:10.1371/journal.pone.0083429.

164. He B, Zhang Y, Kang B, Xiao J, Xie B, Wang Z. Protection of oral hydrogen water as an antioxidant on pulmonary hypertension. Mol Biol Rep. 2013:40(9):5513-21. doi:10.1007/s11033-013-2653-9.

165. Hayashi T, Yoshioka T, Hasegawa K, Miyamura M, Mori T, Ukimura A, et al. Inhalation of hydrogen gas attenuates left ventricular remodeling induced by intermittent hypoxia in mice. Am J Physiol Heart Circ Physiol. 2011:301(3):H1062-9. doi:10.1152/ajpheart.00150.2011.

166. Kato R, Nomura A, Sakamoto A, Yasuda Y, Amatani K, Nagai S, et al. Hydrogen gas attenuates embryonic gene expression and prevents left ventricular remodeling induced by intermittent hypoxia in cardiomyopathic hamsters. Am J Physiol Heart Circ Physiol. 2014;307(11):H1626-33. doi:10.1152/ajpheart.00228.2014 
167. Yu YS, Zheng H. Chronic hydrogen-rich saline treatment reduces oxidative stress and attenuates left ventricular hypertrophy in spontaneous hypertensive rats. Mol Cell Biochem. 2012;365(1-2):233-42. doi:10.1007/s11010-012-1264-4

168. Zhang JY, Wu QF, Wan Y, Song SD, Xu J, Xu XS, et al. Protective role of hydrogen-rich water on aspirin-induced gastric mucosal damage in rats. World J Gastroenterol. 2014;20(6):1614-22. doi:10.3748/wjg.v20.i6.1614.

169. Xue J, Shang G, Tanaka Y, Saihara Y, Hou L, Velasquez N, et al. Dose-dependent inhibition of gastric injury by hydrogen in alkaline electrolyzed drinking water. BMC Complement Altern Med. 2014;14:81. doi:10.1186/1472-6882-14-81

170. Zheng X, Mao Y, Cai J, Li Y, Liu W, Sun P, et al. Hydrogen-rich saline protects against intestinal ischemia/reperfusion injury in rats. Free Radic Res. 2009;43(5):478-84. doi:10.1080/10715760902870603.

171. Chen H, Sun YP, Hu PF, Liu WW, Xiang HG, Li Y, et al. The effects of hydrogen-rich saline on the contractile and structural changes of intestine induced by ischemia-reperfusion in rats. J Surg Res. 2011;167(2):316-22. doi:10.1016/j.jss.2009.07.045

172. Kajiya M, Silva MJ, Sato K, Ouhara K, Kawai T. Hydrogen mediates suppression of colon inflammation induced by dextran sodium sulfate. Biochem Biophys Res Commun. 2009:386(1):11-5. doi:10.1016 j.bbrc.2009.05.117.

173. He J, Xiong S, Zhang J, Wang J, Sun A, Mei X, et al. Protective effects of hydrogen-rich saline on ulcerative colitis rat model. J Surg Res. 2013;185(1):174-81. doi:10.1016/j.jss.2013.05.047.

174. Chen X, Zhai X, Shi J, Liu WW, Tao H, Sun $X$, et al. Lactulose mediates suppression of dextran sodium sulfate-induced colon inflammation by increasing hydrogen production. Dig Dis Sci. 2013;58(6):1560-8. doi:10.1007/s10620-013-2563-7.

175. Sheng Q, Lv Z, Cai W, Song H, Qian L, Wang X. Protective effects of hydrogen-rich saline on necrotizing enterocolitis in neonatal rats. J Pediatr Surg. 2013;48(8):1697-706. doi:10.1016/j.jpedsurg.2012.11.038.

176. Nishimura N, Tanabe H, Sasaki Y, Makita Y, Ohata M, Yokoyama S, et al. Pectin and high-amylose maize starch increase caecal hydrogen production and relieve hepatic ischaemia-reperfusion injury in rats. Br J Nutr. 2012;107(4):485-92. doi:10.1017/S0007114511003229.

177. Liu Y, Yang L, Tao K, Vizcaychipi MP, Lloyd DM, Sun X, et al. Protective effects of hydrogen enriched saline on liver ischemia reperfusion injury by reducing oxidative stress and HMGB1 release. BMC Gastroenterol. 2014;14:12. doi:10.1186/1471-230X-14-12.

178. Matsuno N, Watanabe R, Kimura M, Iwata S, Fujiyama M, Kono S, et al. Beneficial effects of hydrogen gas on porcine liver reperfusion injury with use of total vascular exclusion and active venous bypass. Transplant Proc. 2014;46(4):1104-6. doi:10.1016/j.transproceed.2013.11.134

179. Xia C, Liu W, Zeng D, Zhu L, Sun X, Sun X. Effect of hydrogen-rich water on oxidative stress, liver function, and viral load in patients with chronic hepatitis B. Clin Transl Sci. 2013;6(5):372-5. doi:10.1111/cts.12076.

180. Kawai D, Takaki A, Nakatsuka A, Wada J, Tamaki N, Yasunaka T, et al. Hydrogen-rich water prevents progression of nonalcoholic steatohepatitis and accompanying hepatocarcinogenesis in mice. Hepatology. 2012:56(3):912-21. doi:10.1002/hep.25782.

181. Xiang L, Tan JW, Huang LJ, Jia L, Liu YQ, Zhao YQ, et al. Inhalation of hydrogen gas reduces liver injury during major hepatotectomy in swine. World J Gastroenterol. 2012;18(37):5197-204. doi:10.3748/wjg.v18.i37.5197.

182. Kajiya M, Sato K, Silva MJ, Ouhara K, Do PM, Shanmugam KT, et al. Hydrogen from intestinal bacteria is protective for Concanavalin A-induced hepatitis. Biochem Biophys Res Commun. 2009:386(2):316-21. doi:10.1016/ j.bbrc.2009.06.024

183. Lee PC, Yang YY, Huang CS, Hsieh SL, Lee KC, Hsieh YC, et al. Concomitant inhibition of oxidative stress and angiogenesis by chronic hydrogen-rich saline and $\mathrm{N}$-acetylcysteine treatments improves systemic, splanchnic and hepatic hemodynamics of cirrhotic rats. Hepatol Res. 2015;45(5):578-88. doi:10.1111/hepr.12379.

184. Koyama Y, Taura K, Hatano E, Tanabe K, Yamamoto G, Nakamura K, et al. Effects of oral intake of hydrogen water on liver fibrogenesis in mice. Hepatol Res. 2014;44(6):663-77. doi:10.1111/hepr.12165.

185. Chen H, Sun YP, Li Y, Liu WW, Xiang HG, Fan LY, et al. Hydrogen-rich saline ameliorates the severity of $\mathrm{I}$-arginine-induced acute pancreatitis in rats. Biochem Biophys Res Commun. 2010;393(2):308-13. doi:10.1016/j.bbrc.2010.02.005.

186. Ren J, Luo Z, Tian F, Wang Q, Li K, Wang C. Hydrogen-rich saline reduces the oxidative stress and relieves the severity of trauma-induced acute pancreatitis in rats. J Trauma Acute Care Surg. 2012;72(6):1555-61. doi:10.1097/TA.0b013e31824a7913.

187. Zhang DQ, Feng $H$, Chen WC. Effects of hydrogen-rich saline on taurocholate-induced acute pancreatitis in rat. Evid Based Complement Alternat Med. 2013;2013:731932. doi:10.1155/2013/731932.

188. Zhu WJ, Nakayama M, Mori T, Nakayama K, Katoh J, Murata Y, et al. Intake of water with high levels of dissolved hydrogen $(\mathrm{H} 2)$ suppresses ischemia-induced cardio-renal injury in Dahl salt-sensitive rats. Nephrol Dial Transplant. 2011;26(7):2112-8. doi:10.1093/ndt/gfq727.

189. Shingu C, Koga H, Hagiwara S, Matsumoto S, Goto K, Yokoi I, et al. Hydrogen-rich saline solution attenuates renal ischemia-reperfusion injury. J Anesth. 2010;24(4):569-74. doi:10.1007/s00540-010-0942-1.

190. Wang F, Yu G, Liu SY, Li JB, Wang JF, Bo LL, et al. Hydrogen-rich saline protects against renal ischemia/reperfusion injury in rats. J Surg Res. 2011;167(2):e339-44. doi:10.1016/j.jss.2010.11.005

191. Xu B, Zhang YB, Li ZZ, Yang MW, Wang S, Jiang DP. Hydrogen-rich saline ameliorates renal injury induced by unilateral ureteral obstruction in rats. Int Immunopharmacol. 2013;17(2):447-52. doi:10.1016/j.intimp.2013.06.033.

192. Gu H, Yang M, Zhao X, Zhao B, Sun X, Gao X. Pretreatment with hydrogen-rich saline reduces the damage caused by glycerol-induced rhabdomyolysis and acute kidney injury in rats. J Surg Res. 2014;188(1):243-9. doi:10.1016/j.jss.2013.12.007.

193. Liu W, Dong XS, Sun YQ, Liu Z. A novel fluid resuscitation protocol: provide more protection on acute kidney injury during septic shock in rats. Int J Clin Exp Med. 2014;7(4):919-26.

194. Homma K, Yoshida T, Yamashita M, Hayashida K, Hayashi M, Hori S. Inhalation of Hydrogen Gas Is Beneficial for Preventing ContrastInduced Acute Kidney Injury in Rats. Nephron Exp Nephrol. 2015. doi:10.1159/000369068.

195. Nakashima-Kamimura N, Mori T, Ohsawa I, Asoh S, Ohta S. Molecular hydrogen alleviates nephrotoxicity induced by an anti-cancer drug cisplatin without compromising anti-tumor activity in mice. Cancer Chemother Pharmacol. 2009;64(4):753-61. doi:10.1007/s00280-008-0924-2.

196. Kitamura A, Kobayashi S, Matsushita T, Fujinawa H, Murase K. Experimental verification of protective effect of hydrogen-rich water against cisplatininduced nephrotoxicity in rats using dynamic contrast-enhanced $\mathrm{CT}$. Br J Radiol. 2010;83(990):509-14. doi:10.1259/bjr/25604811.

197. Matsushita T, Kusakabe Y, Kitamura A, Okada S, Murase K. Investigation of protective effect of hydrogen-rich water against cisplatin-induced nephrotoxicity in rats using blood oxygenation level-dependent magnetic resonance imaging. Jpn J Radiol. 2011;29(7):503-12. doi:10.1007/s11604-011-0588-4.

198. Matsushita T, Kusakabe Y, Kitamura A, Okada S, Murase K. Protective effect of hydrogen-rich water against gentamicin-induced nephrotoxicity in rats using blood oxygenation level-dependent MR imaging. Magn Reson Med Sci. 2011;10(3):169-76.

199. Katakura M, Hashimoto M, Tanabe $Y$, Shido O. Hydrogen-rich water inhibits glucose and alpha,beta -dicarbonyl compound-induced reactive oxygen species production in the SHR.Cg-Leprcp/NDmcr rat kidney. Med Gas Res. 2012;2(1):18. doi:10.1186/2045-9912-2-18.

200. Peng Z, Chen W, Wang L, Ye Z, Gao S, Sun X, et al. Inhalation of hydrogen gas ameliorates glyoxylate-induced calcium oxalate deposition and renal oxidative stress in mice. Int J Clin Exp Pathol. 2015;8(3):2680-9.

201. Matsumoto S, Ueda T, Kakizaki H. Effect of supplementation with hydrogenrich water in patients with interstitial cystitis/painful bladder syndrome. Urology. 2013;81(2):226-30. doi:10.1016/j.urology.2012.10.026.

202. Lee JW, Kim JI, Lee YA, Lee DH, Song CS, Cho YJ, et al. Inhaled hydrogen gas therapy for prevention of testicular ischemia/reperfusion injury in rats. J Pediatr Surg. 2012;47(4):736-42. doi:10.1016/j.jpedsurg.2011.09.035.

203. Jiang $D$, Wu D, Zhang $Y, X u B$, Sun $X, L i$ Z. Protective effects of hydrogen rich saline solution on experimental testicular ischemia-reperfusion injury in rats. J Urol. 2012;187(6):2249-53. doi:10.1016/j.juro.2012.01.029.

204. Fan M, Xu X, He X, Chen L, Qian L, Liu J, et al. Protective effects of hydrogenrich saline against erectile dysfunction in a streptozotocin induced diabetic rat model. J Urol. 2013;190(1):350-6. doi:10.1016/j.juro.2012.12.001.

205. Li S, Lu D, Zhang Y, Zhang Y. Long-term treatment of hydrogen-rich saline abates testicular oxidative stress induced by nicotine in mice. J Assist Reprod Genet. 2014;31(1):109-14. doi:10.1007/s10815-013-0102-2.

206. Chen S, Jiang W. Effect of hydrogen injected subcutaneously on testicular tissues of rats exposed to cigarette smoke. Int J Clin Exp Med. 2015;8(4):5565-70 
207. Zhao L, Wang YB, Qin SR, Ma XM, Sun XJ, Wang ML, et al. Protective effect of hydrogen-rich saline on ischemia/reperfusion injury in rat skin flap. J Zhejiang Univ Sci B. 2013;14(5):382-91. doi:10.1631/jzus.B1200317.

208. Yoon KS, Huang XZ, Yoon YS, Kim SK, Song SB, Chang BS, et al. Histological study on the effect of electrolyzed reduced water-bathing on UVB radiation-induced skin injury in hairless mice. Biol Pharm Bull. 2011;34(11):1671-7.

209. Guo Z, Zhou B, Li W, Sun X, Luo D. Hydrogen-rich saline protects against ultraviolet B radiation injury in rats. J Biomed Res. 2012;26(5):365-71. doi:10.7555/JBR.26.20110037.

210. Kato S, Saitoh Y, Iwai K, Miwa N. Hydrogen-rich electrolyzed warm water represses wrinkle formation against UVA ray together with type-I collagen production and oxidative-stress diminishment in fibroblasts and cell-injury prevention in keratinocytes. J Photochem Photobiol B. 2012;106:24-33. doi:10.1016/j.jphotobiol.2011.09.006.

211. Ignacio RM, Yoon Y-S, Sajo MEJ, Kim C-S, Kim D-H, Kim S-K, et al. The balneotherapy effect of hydrogen reduced water on UVB-mediated skin injury in hairless mice. Mol Cell Toxicol. 2013;9(1):15-21. doi:10.1007/ s13273-013-0003-6.

212. Ono H, Nishijima Y, Adachi N, Sakamoto M, Kudo Y, Nakazawa J, et al. Hydrogen $\left(\mathrm{H}_{2}\right)$ treatment for acute erythymatous skin diseases. A report of 4 patients with safety data and a non-controlled feasibility study with $\mathrm{H} 2$ concentration measurement on two volunteers. Med Gas Res. 2012;2(1):14. doi:10.1186/2045-9912-2-14

213. Ignacio RM, Kwak HS, Yun YU, Sajo ME, Yoon YS, Kim CS, et al. The Drinking Effect of Hydrogen Water on Atopic Dermatitis Induced by Dermatophagoides farinae Allergen in NC/Nga Mice. Evid Based Complement Alternat Med. 2013;2013:538673. doi:10.1155/2013/538673.

214. Yoon YS, Sajo ME, Ignacio RM, Kim SK, Kim CS, Lee KJ. Positive Effects of hydrogen water on 2,4-dinitrochlorobenzene-induced atopic dermatitis in NC/Nga mice. Biol Pharm Bull. 2014;37(9):1480-5.

215. Ishibashi T, Ichikawa M, Sato B, Shibata S, Hara Y, Naritomi Y, et al. Improvement of psoriasis-associated arthritis and skin lesions by treatment with molecular hydrogen: A report of three cases. Mol Med Rep. 2015;12(2):2757-64. doi:10.3892/mmr.2015.3707.

216. Li Q, Kato S, Matsuoka D, Tanaka H, Miwa N. Hydrogen water intake via tube-feeding for patients with pressure ulcer and its reconstructive effects on normal human skin cells in vitro. Med Gas Res. 2013;3(1):20. doi:10.1186/2045-9912-3-20.

217. Yu W, Chiu Y, Lee C, Yoshioka T, Yu H. Hydrogen-enriched water restoration of impaired calcium propagation by arsenic in primary keratinocytes. J Asian Earth Sci. 2013;77:342-8. doi:10.1016/j.jseaes.2013.07.007.

218. Ishibashi T, Sato B, Rikitake M, Seo T, Kurokawa R, Hara Y, et al. Consumption of water containing a high concentration of molecular hydrogen reduces oxidative stress and disease activity in patients with rheumatoid arthritis: an open-label pilot study. Med Gas Res. 2012;2(1):27. doi:10.1186/2045-9912-2-27.

219. Ishibashi T, Sato B, Shibata S, Sakai T, Hara Y, Naritomi Y, et al. Therapeutic efficacy of infused molecular hydrogen in saline on rheumatoid arthritis: a randomized, double-blind, placebo-controlled pilot study. Int Immunopharmacol. 2014;21(2):468-73. doi:10.1016/j.intimp.2014.06.001.

220. Cai WW, Zhang MH, Yu YS, Cai JH. Treatment with hydrogen molecule alleviates TNFalpha-induced cell injury in osteoblast. Mol Cell Biochem. 2013;373(1-2):1-9. doi:10.1007/s11010-012-1450-4.

221. Hanaoka T, Kamimura N, Yokota T, Takai S, Ohta S. Molecular hydrogen protects chondrocytes from oxidative stress and indirectly alters gene expressions through reducing peroxynitrite derived from nitric oxide. Med Gas Res. 2011;1(1):18. doi:10.1186/2045-9912-1-18.

222. Huang T, Wang W, Tu C, Yang Z, Bramwell D, Sun X. Hydrogen-rich saline attenuates ischemia-reperfusion injury in skeletal muscle. J Surg Res. 2015;194(2):471-80. doi:10.1016/j.jss.2014.12.016.

223. Ito M, Ibi T, Sahashi K, Ichihara M, Ito M, Ohno K. Open-label trial and randomized, double-blind, placebo-controlled, crossover trial of hydrogenenriched water for mitochondrial and inflammatory myopathies. Med Gas Res. 2011;1(1):24. doi:10.1186/2045-9912-1-24.

224. Aoki K, Nakao A, Adachi T, Matsui Y, Miyakawa S. Pilot study: Effects of drinking hydrogen-rich water on muscle fatigue caused by acute exercise in elite athletes. Med Gas Res. 2012;2(1):12. doi:10.1186/2045-9912-2-12.

225. Ostojic SM, Vukomanovic B, Calleja-Gonzalez J, Hoffman JR. Effectiveness of oral and topical hydrogen for sports-related soft tissue injuries. Postgrad Med. 2014;126(5):187-95. doi:10.3810/pgm.2014.09.2813.
226. Ohsawa I, Nishimaki K, Yamagata K, Ishikawa M, Ohta S. Consumption of hydrogen water prevents atherosclerosis in apolipoprotein E knockout mice. Biochem Biophys Res Commun. 2008;377(4):1195-8. doi:10.1016/ j.bbrc.2008.10.156.

227. Ekuni D, Tomofuji T, Endo Y, Kasuyama K, Irie K, Azuma T, et al. Hydrogenrich water prevents lipid deposition in the descending aorta in a rat periodontitis model. Arch Oral Biol. 2012;57(12):1615-22. doi:10.1016/ j.archoralbio.2012.04.013.

228. Jiang H, Yu P, Qian DH, Qin ZX, Sun XJ, Yu J, et al. Hydrogen-rich medium suppresses the generation of reactive oxygen species, elevates the $\mathrm{Bcl}-2 / \mathrm{Bax}$ ratio and inhibits advanced glycation end product-induced apoptosis. Int J Mol Med. 2013;31(6):1381-7. doi:10.3892/ijmm.2013.1334.

229. Sun Q, Kawamura T, Masutani K, Peng X, Sun Q, Stolz DB, et al. Oral intake of hydrogen-rich water inhibits intimal hyperplasia in arterialized vein grafts in rats. Cardiovasc Res. 2012;94(1):144-53. doi:10.1093/cvr/Cvs024.

230. Sakai T, Sato B, Hara K, Hara Y, Naritomi Y, Koyanagi S, et al. Consumption of water containing over $3.5 \mathrm{mg}$ of dissolved hydrogen could improve vascular endothelial function. Vasc Health Risk Manag. 2014;10:591-7. doi:10.2147/NHRM.S68844.

231. Zhao S, Mei K, Qian L, Yang Y, Liu W, Huang Y, et al. Therapeutic effects of hydrogen-rich solution on aplastic anemia in vivo. Cell Physiol Biochem. 2013;32(3):549-60. doi:10.1159/000354459.

232. Kawasaki H, Guan J, Tamama K. Hydrogen gas treatment prolongs replicative lifespan of bone marrow multipotential stromal cells in vitro while preserving differentiation and paracrine potentials. Biochem Biophys Res Commun. 2010;397(3):608-13. doi:10.1016/j.bbrc.2010.06.009.

233. Tanikawa R, Takahashi I, Okubo N, Ono M, Okumura T, Ishibashi G, et al. Relationship between Exhaled Hydrogen and Human Neutrophil Function in the Japanese General Population. Hirosaki Medical Journal. 2015;65:138-46

234. Takeuchi S, Wada K, Nagatani K, Osada H, Otani N, Nawashiro H. Hydrogen may inhibit collagen-induced platelet aggregation: an ex vivo and in vivo study. Intern Med. 2012;51(11):1309-13.

235. Kato S, Hokama R, Okayasu H, Saitoh Y, Iwai K, Miwa N. Colloidal platinum in hydrogen-rich water exhibits radical-scavenging activity and improves blood fluidity. J Nanosci Nanotechnol. 2012;12(5):4019-27.

236. Kajiyama S, Hasegawa G, Asano M, Hosoda H, Fukui M, Nakamura N, et al. Supplementation of hydrogen-rich water improves lipid and glucose metabolism in patients with type 2 diabetes or impaired glucose tolerance. Nutr Res. 2008;28(3):137-43. doi:10.1016/j.nutres.2008.01.008.

237. Kamimura N, Nishimaki K, Ohsawa I, Ohta S. Molecular hydrogen improves obesity and diabetes by inducing hepatic FGF21 and stimulating energy metabolism in $\mathrm{db} / \mathrm{db}$ mice. Obesity. 2011;19(7):1396-403. doi:10.1038/oby.2011.6.

238. Li Y, Hamasaki T, Nakamichi N, Kashiwagi T, Komatsu T, Ye J, et al. Suppressive effects of electrolyzed reduced water on alloxan-induced apoptosis and type 1 diabetes mellitus. Cytotechnology. 2011;63(2):119-31. doi:10.1007/s10616-010-9317-6.

239. Yu P, Wang Z, Sun X, Chen X, Zeng S, Chen L, et al. Hydrogen-rich medium protects human skin fibroblasts from high glucose or mannitol induced oxidative damage. Biochem Biophys Res Commun. 2011;409(2):350-5. doi:10.1016/j.bbrc.2011.05.024.

240. Wang QJ, Zha XJ, Kang ZM, Xu MJ, Huang Q, Zou DJ. Therapeutic effects of hydrogen saturated saline on rat diabetic model and insulin resistant model via reduction of oxidative stress. Chin Med J (Engl). 2012;125(9):1633-7. doi:10.3760/cma.j.issn.0366-6999.2012.09.020.

241. Amitani H, Asakawa A, Cheng K, Amitani M, Kaimoto K, Nakano M, et al. Hydrogen improves glycemic control in type1 diabetic animal model by promoting glucose uptake into skeletal muscle. PLoS One. 2013;8(1):e53913. doi:10.1371/journal.pone.0053913.

242. Zong C, Song G, Yao S, Li L, Yu Y, Feng L, et al. Administration of hydrogensaturated saline decreases plasma low-density lipoprotein cholesterol levels and improves high-density lipoprotein function in high-fat diet-fed hamsters. Metabolism. 2012;61(6):794-800. doi:10.1016/ j.metabol.2011.10.014.

243. Song G, Li M, Sang H, Zhang L, Li X, Yao S, et al. Hydrogen-rich water decreases serum LDL-cholesterol levels and improves HDL function in patients with potential metabolic syndrome. J Lipid Res. 2013;54(7):1884-93. doi:10.1194/jlr.M036640.

244. Song G, Lin Q, Zhao H, Liu M, Ye F, Sun Y, et al. Hydrogen activates ATPbinding cassette transporter A1-dependent efflux ex vivo and improves 
high-density lipoprotein function in patients with hypercholesterolemia: a double-blinded, randomized and placebo-controlled trial. J Clin Endocrinol Metab. 2015;100:2724-33. doi:10.1210/jc.2015-1321.

245. Nakao A, Toyoda Y, Sharma P, Evans M, Guthrie N. Effectiveness of hydrogen rich water on antioxidant status of subjects with potential metabolic syndrome-an open label pilot study. J Clin Biochem Nutr. 2010;46(2):140-9. doi:10.3164/jcbn.09-100.

246. Hashimoto M, Katakura M, Nabika T, Tanabe Y, Hossain S, Tsuchikura S, et al. Effects of hydrogen-rich water on abnormalities in a SHR.Cg-Leprcp/NDmcr rat - a metabolic syndrome rat model. Med Gas Res. 2011;1 (1):26. doi:10.1186/2045-9912-1-26

247. Nishimura N, Tanabe H, Adachi M, Yamamoto T, Fukushima M. Colonic hydrogen generated from fructan diffuses into the abdominal cavity and reduces adipose mRNA abundance of cytokines in rats. J Nutr. 2013;143(12):1943-9. doi:10.3945/jn.113.183004.

248. Nakai Y, Sato B, Ushiama S, Okada S, Abe K, Arai S. Hepatic oxidoreductionrelated genes are upregulated by administration of hydrogen-saturated drinking water. Biosci Biotechnol Biochem. 2011;75(4):774-6. doi:10.1271/ bbb.100819.

249. Ostojic SM. Serum alkalinization and hydrogen-rich water in healthy men. Mayo Clin Proc. 2012;87(5):501-2. doi:10.1016/.mayocp.2012.02.008.

250. Ostojic SM, Stojanovic MD. Hydrogen-rich water affected blood alkalinity in physically active men. Res Sports Med. 2014;22(1):49-60. doi:10.1080/ 15438627.2013.852092.

251. Xie K, Yu Y, Pei Y, Hou L, Chen S, Xiong L, et al. Protective effects of hydrogen gas on murine polymicrobial sepsis via reducing oxidative stress and HMGB1 release. Shock. 2010;34(1):90-7. doi:10.1097/ SHK.0b013e3181cdc4ae.

252. Zhou J, Chen Y, Huang GQ, Li J, Wu GM, Liu L, et al. Hydrogen-rich saline reverses oxidative stress, cognitive impairment, and mortality in rats submitted to sepsis by cecal ligation and puncture. J Surg Res. 2012;178(1):390-400. doi:10.1016/j.jss.2012.01.041.

253. Xie K, Fu W, Xing W, Li A, Chen H, Han H, et al. Combination therapy with molecular hydrogen and hyperoxia in a murine model of polymicrobial sepsis. Shock. 2012;38(6):656-63. doi:10.1097/SHK.0b013e3182758646.

254. Li GM, Ji MH, Sun XJ, Zeng OT, Tian M, Fan YX, et al. Effects of hydrogen-rich saline treatment on polymicrobial sepsis. J Surg Res. 2013;181(2):279-86. doi:10.1016/j.jss.2012.06.058.

255. Liu W, Shan LP, Dong XS, Liu XW, Ma T, Liu Z. Combined early fluid resuscitation and hydrogen inhalation attenuates lung and intestine injury. World J Gastroenterol. 2013;19(4):492-502. doi:10.3748/wjg.v19.44.492.

256. Yu Y, Wang WN, Han HZ, Xie KL, Wang GL, Yu YH. Protective effects of hydrogen-rich medium on lipopolysaccharide-induced monocytic adhesion and vascular endothelial permeability through regulation of vascular endothelial cadherin. Genet Mol Res. 2015:14(2):6202-12. doi:10.4238/ 2015.June.9.6.

257. Xie K, Yu Y, Zhang Z, Liu W, Pei Y, Xiong L, et al. Hydrogen gas improves survival rate and organ damage in zymosan-induced generalized inflammation model. Shock. 2010;34(5):495-501. doi:10.1097/ SHK.0b013e3181def9aa.

258. Xu Z, Zhou J, Cai J, Zhu Z, Sun X, Jiang C. Anti-inflammation effects of hydrogen saline in LPS activated macrophages and carrageenan induced paw oedema. J Inflamm (Lond). 2012;9:2. doi:10.1186/1476-9255-9-2.

259. Fujii $Y$, Shirai M, Inamori S, Shimouchi A, Sonobe T, Tsuchimochi H, et al. Insufflation of hydrogen gas restrains the inflammatory response of cardiopulmonary bypass in a rat model. Artif Organs. 2013;37(2):136-41. doi:10.1111/j.1525-1594.2012.01535.x.

260. Cai J, Kang Z, Liu WW, Luo X, Qiang S, Zhang JH, et al. Hydrogen therapy reduces apoptosis in neonatal hypoxia-ischemia rat model. Neurosci Lett. 2008;441 (2):167-72. doi:10.1016/j.neulet.2008.05.077.

261. Cai J, Kang Z, Liu K, Liu W, Li R, Zhang JH, et al. Neuroprotective effects of hydrogen saline in neonatal hypoxia-ischemia rat model. Brain Res. 2009;1256:129-37. doi:10.1016/j.brainres.2008.11.048.

262. Domoki F, Olah O, Zimmermann A, Nemeth I, Toth-Szuki V, Hugyecz M, et al. Hydrogen is neuroprotective and preserves cerebrovascular reactivity in asphyxiated newborn pigs. Pediatr Res. 2010;68(5):387-92. doi:10.1203/ PDR.0b013e3181f2e81c

263. Mano Y, Kotani T, Ito M, Nagai T, Ichinohashi Y, Yamada K, et al. Maternal molecular hydrogen administration ameliorates rat fetal hippocampal damage caused by in utero ischemia-reperfusion. Free Radic Biol Med. 2014;69:324-30. doi:10.1016/j.freeradbiomed.2014.01.037.
264. Yang $X$, Guo L, Sun $X$, Chen $X$, Tong $X$. Protective effects of hydrogen-rich saline in preeclampsia rat model. Placenta. 2011;32(9):681-6. doi:10.1016/ j.placenta.2011.06.020.

265. Guan Z, Li HF, Guo LL, Yang X. Effects of vitamin C, vitamin E, and molecular hydrogen on the placental function in trophoblast cells. Arch Gynecol Obstet. 2015;292(2):337-42. doi:10.1007/s00404-015-3647-8.

266. Saitoh Y, Okayasu H, Xiao L, Harata Y, Miwa N. Neutral pH hydrogenenriched electrolyzed water achieves tumor-preferential clonal growth inhibition over normal cells and tumor invasion inhibition concurrently with intracellular oxidant repression. Oncol Res. 2008;17(6):247-55.

267. Zhao L, Zhou C, Zhang J, Gao F, Li B, Chuai Y, et al. Hydrogen protects mice from radiation induced thymic lymphoma in BALB/C mice. Int J Biol Sci. 2011;7(3):297-300.

268. Ye J, Li Y, Hamasaki T, Nakamichi N, Komatsu T, Kashiwagi T, et al. Inhibitory effect of electrolyzed reduced water on tumor angiogenesis. Biol Pharm Bull. 2008;31(1):19-26.

269. Runtuwene J, Amitani H, Amitani M, Asakawa A, Cheng KC, Inui A. Hydrogen-water enhances 5-fluorouracil-induced inhibition of colon cancer. PeerJ. 2015;3:e859. doi:10.7717/peeri.859.

270. Qian L, Cao F, Cui J, Wang Y, Huang Y, Chuai Y, et al. The potential cardioprotective effects of hydrogen in irradiated mice. J Radiat Res. 2010;51(6):741-7. doi:10.1269/jr.10093.

271. Terasaki Y, Ohsawa I, Terasaki M, Takahashi M, Kunugi S, Dedong K, et al. Hydrogen therapy attenuates irradiation-induced lung damage by reducing oxidative stress. Am J Physiol Lung Cell Mol Physiol. 2011;301(4):L415-26. doi:10.1152/ajplung.00008.2011.

272. Jiang Z, Xu B, Yang M, Li Z, Zhang Y, Jiang D. Protection by hydrogen against gamma ray-induced testicular damage in rats. Basic Clin Pharmacol Toxicol. 2013;112(3):186-91. doi:10.1111/bcpt.12016.

273. Mei K, Zhao S, Qian L, Li B, Ni J, Cai J. Hydrogen protects rats from dermatitis caused by local radiation. J Dermatolog Treat. 2014;25(2):182-8. doi:10.3109/09546634.2012.762639.

274. Watanabe S, Fujita M, Ishihara M, Tachibana S, Yamamoto Y, Kaji T, et al. Protective effect of inhalation of hydrogen gas on radiation-induced dermatitis and skin injury in rats. J Radiat Res. 2014;55(6):1107-13. doi:10.1093/jrr/rru067.

275. Qian L, Cao F, Cui J, Huang Y, Zhou X, Liu S, et al. Radioprotective effect of hydrogen in cultured cells and mice. Free Radic Res. 2010:44(3):275-82. doi:10.3109/10715760903468758.

276. Qian L, Li B, Cao F, Huang Y, Liu S, Cai J, et al. Hydrogen-rich PBS protects cultured human cells from ionizing radiation-induced cellular damage. Nucl Technol Radiat Prot. 2010;25(1):23-9. doi:10.2298/ntrp1001023q.

277. Chuai Y, Gao F, Li B, Zhao L, Qian L, Cao F, et al. Hydrogen-rich saline attenuates radiation-induced male germ cell loss in mice through reducing hydroxyl radicals. Biochem J. 2012;442(1):49-56. doi:10.1042/BJ20111786.

278. Yang Y, Li B, Liu C, Chuai Y, Lei J, Gao F, et al. Hydrogen-rich saline protects immunocytes from radiation-induced apoptosis. Med Sci Monit. 2012;18(4):BR144-8.

279. Chuai Y, Shen J, Qian L, Wang Y, Huang Y, Gao F, et al. Hydrogen-rich saline protects spermatogenesis and hematopoiesis in irradiated BALB/C mice. Med Sci Monit. 2012;18(3):BR89-94.

280. Yang Y, Gao F, Zhang H, Hunag Y, Zhang P, Liu C, et al. Molecular hydrogen protects human lymphocyte $\mathrm{AHH}-1$ cells against $12 \mathrm{C} 6+$ heavy ion radiation. Int J Radiat Biol. 2013;89(12):1003-8. doi:10.3109/09553002.2013.817704.

281. Kang KM, Kang YN, Choi IB, Gu Y, Kawamura T, Toyoda Y, et al. Effects of drinking hydrogen-rich water on the quality of life of patients treated with radiotherapy for liver tumors. Med Gas Res. 2011;1(1):11. doi:10.1186/2045-9912-1-11.

282. Zhao S, Yang Y, Liu W, Xuan Z, Wu S, Yu S, et al. Protective effect of hydrogen-rich saline against radiation-induced immune dysfunction. J Cell Mol Med. 2014;18(5):938-46. doi:10.1111/jcmm.12245.

283. Sun Q, Cai J, Zhou J, Tao H, Zhang JH, Zhang W, et al. Hydrogen-rich saline reduces delayed neurologic sequelae in experimental carbon monoxide toxicity. Crit Care Med. 2011;39(4):765-9. doi:10.1097/ CCM.0b013e318206bf44.

284. Wang W, Li Y, Ren J, Xia F, Li J, Zhang Z. Hydrogen rich saline reduces immune-mediated brain injury in rats with acute carbon monoxide poisoning Neurol Res. 2012;34(10):1007-15. doi:10.1179/1743132812Y.0000000106.

285. Shen MH, Cai JM, Sun Q, Zhang DW, Huo ZL, He J, et al. Neuroprotective effect of hydrogen-rich saline in acute carbon monoxide poisoning. CNS Neurosci Ther. 2013;19(5):361-3. doi:10.1111/cns.12094. 
286. Wang W, Tian L, Li Y, Wang X, Xia F, Li L, et al. Effects of hydrogen-rich saline on rats with acute carbon monoxide poisoning. J Emerg Med. 2013;44(1):107-15. doi:10.1016/j.jemermed.2012.01.065.

287. Yonamine R, Satoh Y, Kodama M, Araki Y, Kazama T. Coadministration of hydrogen gas as part of the carrier gas mixture suppresses neuronal apoptosis and subsequent behavioral deficits caused by neonatal exposure to sevoflurane in mice. Anesthesiology. 2013;118(1):105-13. doi:10.1097/ALN.0b013e318275146d.

288. Takaenoki Y, Satoh Y, Araki Y, Kodama M, Yonamine R, Yufune S, et al. Neonatal exposure to sevoflurane in mice causes deficits in maternal behavior later in adulthood. Anesthesiology. 2014;120(2):403-15. doi:10.1097/ALN.0000435846.28299.e7.

289. Wu S, Zhu L, Yang J, Fan Z, Dong Y, Luan R, et al. Hydrogen-containing saline attenuates doxorubicin-induced heart failure in rats. Pharmazie. 2014;69(8):633-6.

290. Yoon YS, Kim DH, Kim SK, Song SB, Uh Y, Jin D, et al. The melamine excretion effect of the electrolyzed reduced water in melamine-fed mice. Food Chem Toxicol. 2011;49(8):1814-9. doi:10.1016/j.fct.2011.04.033.

291. Wang T, Zhao L, Liu M, Xie F, Ma X, Zhao P, et al. Oral intake of hydrogen-rich water ameliorated chlorpyrifos-induced neurotoxicity in rats. Toxicol Appl Pharmacol. 2014;280(1):169-76. doi:10.1016/ j.taap.2014.06.011.

292. Nakao A, Kaczorowski DJ, Wang Y, Cardinal JS, Buchholz BM, Sugimoto R, et al. Amelioration of rat cardiac cold ischemia/reperfusion injury with inhaled hydrogen or carbon monoxide, or both. J Heart Lung Transplant. 2010;29(5):544-53. doi:10.1016/j.healun.2009.10.011

293. Tan M, Sun X, Guo L, Su C, Sun X, Xu Z. Hydrogen as additive of HTK solution fortifies myocardial preservation in grafts with prolonged cold ischemia. Int J Cardiol. 2013;167(2):383-90. doi:10.1016/j.ijcard.2011.12.109.

294. Noda K, Tanaka Y, Shigemura N, Kawamura T, Wang Y, Masutani K, et al. Hydrogen-supplemented drinking water protects cardiac allografts from inflammation-associated deterioration. Transpl Int. 2012;25(12):1213-22. doi:10.1111/j.1432-2277.2012.01542.x.

295. Kawamura T, Huang CS, Tochigi N, Lee S, Shigemura N, Billiar TR, et al. Inhaled hydrogen gas therapy for prevention of lung transplant-induced ischemia/reperfusion injury in rats. Transplantation. 2010;90(12):1344-51. doi:10.1097/TP.0b013e3181fe1357.

296. Zhou H, Fu Z, Wei Y, Liu J, Cui X, Yang W, et al. Hydrogen inhalation decreases lung graft injury in brain-dead donor rats. J Heart Lung Transplant. 2013;32(2):251-8. doi:10.1016/j.healun.2012.11.007.

297. Noda K, Shigemura N, Tanaka Y, Bhama J, D'Cunha J, Kobayashi H, et al. Hydrogen preconditioning during ex vivo lung perfusion improves the quality of lung grafts in rats. Transplantation. 2014;98(5):499-506. doi:10.1097/TP.0000000000000254.

298. Haam S, Lee S, Paik HC, Park MS, Song JH, Lim BJ, et al. The effects of hydrogen gas inhalation during ex vivo lung perfusion on donor lungs obtained after cardiac deathdagger. Eur J Cardiothorac Surg. 2015;48:542-7. doi:10.1093/ejcts/ezv057.

299. Liu R, Fang X, Meng C, Xing J, Liu J, Yang W, et al. Lung inflation with hydrogen during the cold ischemia phase decreases lung graft injury in rats. Exp Biol Med (Maywood). 2015;240:1214-22. doi:10.1177/ 1535370214563895.

300. Buchholz BM, Kaczorowski DJ, Sugimoto R, Yang R, Wang Y, Billiar TR, et al. Hydrogen inhalation ameliorates oxidative stress in transplantation induced intestinal graft injury. Am J Transplant. 2008;8(10):2015-24. doi:10.1111/ j.1600-6143.2008.02359.x

301. Shigeta T, Sakamoto S, Li XK, Cai S, Liu C, Kurokawa R, et al. Luminal injection of hydrogen-rich solution attenuates intestinal ischemiareperfusion injury in rats. Transplantation. 2015;99(3):500-7. doi:10.1097/ TP.0000000000000510.

302. Luo ZL, Cheng L, Ren JD, Fang C, Xiang K, Xu HT, et al. Hydrogen-rich saline protects against ischemia/reperfusion injury in grafts after pancreas transplantations by reducing oxidative stress in rats. Mediators Inflamm. 2015;2015:281985. doi:10.1155/2015/281985.

303. Yamada T, Uchida K, Onuma K, Kuzuno J, Ujihira M, Inoue G, et al. Hydrogen supplementation of preservation solution improves viability of osteochondral grafts. ScientificWorldJournal. 2014;2014:109876. doi:10.1155/ 2014/109876.

304. Qian L, Mei K, Shen J, Cai J. Administration of hydrogen-rich saline protects mice from lethal acute graft-versus-host disease (aGVHD). Transplantation. 2013:95(5):658-62. doi:10.1097/TP.0b013e31827e6b23.
305. Yuan L, Chen X, Qian L, Shen J, Cai J. Administration of hydrogen-rich saline in mice with allogeneic hematopoietic stem-cell transplantation. Med Sci Monit. 2015;21:749-54. doi:10.12659/MSM.891338.

306. Hayashida K, Sano M, Kamimura N, Yokota T, Suzuki M, Ohta S, et al. Hydrogen inhalation during normoxic resuscitation improves neurological outcome in a rat model of cardiac arrest independently of targeted temperature management. Circulation. 2014;130(24):2173-80. doi:10.1161/ CIRCULATIONAHA.114.011848.

307. Huo TT, Zeng Y, Liu XN, Sun L, Han HZ, Chen HG, et al. Hydrogen-rich saline improves survival and neurological outcome after cardiac arrest and cardiopulmonary resuscitation in rats. Anesth Analg. 2014;119(2):368-80. doi:10.1213/ANE.0000000000000303.

308. Du Z, Jia H, Liu J, Zhao X, Wang Y, Sun X. Protective effects of hydrogenrich saline in uncontrolled hemorrhagic shock. Exp Ther Med. 2014;7(5):1253-8. doi:10.3892/etm.2014.1572

309. Du Z, Jia H, Liu J, Zhao X, Xu W. Effects of three hydrogen-rich liquids on hemorrhagic shock in rats. J Surg Res. 2015;193(1):377-82. doi:10.1016/ j.jss.2014.06.051.

310. Nakayama M, Kabayama S, Nakano H, Zhu WJ, Terawaki H, Nakayama K, et al. Biological effects of electrolyzed water in hemodialysis. Nephron Clin Pract. 2009:112(1):c9-15. doi:10.1159/000210569.

311. Nakayama M, Nakano H, Hamada H, Itami N, Nakazawa R, Ito S. A novel bioactive haemodialysis system using dissolved dihydrogen $\left(\mathrm{H}_{2}\right)$ produced by water electrolysis: a clinical trial. Nephrol Dial Transplant. 2010;25(9):3026-33. doi:10.1093/ndt/gfa196.

312. Terawaki H, Zhu WJ, Matsuyama Y, Terada T, Takahashi Y, Sakurai K, et al. Effect of a hydrogen ( $\mathrm{H} 2)$-enriched solution on the albumin redox of hemodialysis patients. Hemodial Int. 2014;18(2):459-66. doi:10.1111/hdi.12112

313. Tange Y, Takesawa S, Yoshitake S. Dialysate with high dissolved hydrogen facilitates dissociation of indoxyl sulfate from albumin. Nephrourol Mon. 2015;7(2):e26847. doi:10.5812/numonthly.26847.

314. Terawaki H, Hayashi Y, Zhu WJ, Matsuyama Y, Terada T, Kabayama S, et al. Transperitoneal administration of dissolved hydrogen for peritoneal dialysis patients: a novel approach to suppress oxidative stress in the peritoneal cavity. Med Gas Res. 2013;3(1):14. doi:10.1186/2045-9912-3-14.

315. Terawaki H, Nakano H, Zhu WJ, Nakayama M. Successful treatment of encapsulating peritoneal sclerosis by hemodialysis and peritoneal lavage using dialysate containing dissolved hydrogen. Perit Dial Int. 2015;35(1):107-12. doi:10.3747/pdi.2013.00255.

316. Yan H, Tian H, Kinjo T, Hamasaki T, Tomimatsu K, Nakamichi $N$, et al. Extension of the lifespan of Caenorhabditis elegans by the use of electrolyzed reduced water. Biosci Biotechnol Biochem. 2010;74(10):2011-5. doi:10.1271/bbb.100250.

317. Nakata K, Yamashita N, Noda Y, Ohsawa I. Stimulation of human damaged sperm motility with hydrogen molecule. Med Gas Res. 2015;5(1):2. doi:10.1186/s13618-014-0023-X.

318. Ni XX, Cai ZY, Fan DF, Liu Y, Zhang RJ, Liu SL, et al. Protective effect of hydrogen-rich saline on decompression sickness in rats. Aviat Space Environ Med. 2011:82(6):604-9. doi:10.3357/asem.2964.2011.

319. Saitoh Y, Harata Y, Mizuhashi F, Nakajima M, Miwa N. Biological safety of neutral-pH hydrogen-enriched electrolyzed water upon mutagenicity, genotoxicity and subchronic oral toxicity. Toxicol Ind Health. 2010;26(4):203-16. doi:10.1177/0748233710362989.

320. Chen M, Cui W, Zhu K, Xie Y, Zhang C, Shen W. Hydrogen-rich water alleviates aluminum-induced inhibition of root elongation in alfalfa via decreasing nitric oxide production. J Hazard Mater. 2014;267:40-7. doi:10.1016/j.jhazmat.2013.12.029.

321. Xie $Y$, Mao $Y$, Lai $D$, Zhang $W$, Shen W. H(2) enhances arabidopsis salt tolerance by manipulating ZAT10/12-mediated antioxidant defence and controlling sodium exclusion. PLoS One. 2012;7(11):e49800. doi:10.1371/ journal.pone.0049800.

322. Xu S, Zhu S, Jiang Y, Wang N, Wang R, Shen W, et al. Hydrogen-rich water alleviates salt stress in rice during seed germination. Plant Soil. 2013:370(1-2):47-57. doi:10.1007/s11104-013-1614-3.

323. Hu H, Li P, Wang Y, Gu R. Hydrogen-rich water delays postharvest ripening and senescence of kiwifruit. Food Chem. 2014;156:100-9. doi:10.1016/ j.foodchem.2014.01.067.

324. Xie Y, Mao Y, Zhang W, Lai D, Wang Q, Shen W. Reactive Oxygen SpeciesDependent Nitric Oxide Production Contributes to Hydrogen-Promoted Stomatal Closure in Arabidopsis. Plant Physiol. 2014;165(2):759-73. doi:10.1104/pp. 114.237925 
325. Su N, Wu Q, Liu Y, Cai J, Shen W, Xia K, et al. Hydrogen-rich water reestablishes ROS homeostasis but exerts differential effects on anthocyanin synthesis in two varieties of radish sprouts under UV-A irradiation. J Agric Food Chem. 2014;62(27):6454-62. doi:10.1021/jf5019593.

326. Zhang X, Zhao X, Wang Z, Shen W, Xu X. Protective effects of hydrogen-rich water on the photosynthetic apparatus of maize seedlings (Zea mays L.) as a result of an increase in antioxidant enzyme activities under high light stress. Plant Growth Regul. 2015;77:43-56.

327. Zeng J, Zhang M, Sun X. Molecular hydrogen is involved in phytohormone signaling and stress responses in plants. PLoS One. 2013;8(8):e71038. doi:10.1371/journal.pone.0071038.

328. Jin Q, Zhu K, Cui W, Xie Y, Han B, Shen W. Hydrogen gas acts as a novel bioactive molecule in enhancing plant tolerance to paraquat-induced oxidative stress via the modulation of heme oxygenase- 1 signalling system. Plant Cell Environ. 2013;36(5):956-69. doi:10.1111/pce.12029.

329. Cui W, Gao C, Fang P, Lin G, Shen W. Alleviation of cadmium toxicity in Medicago sativa by hydrogen-rich water. J Hazard Mater. 2013;260:715-24. doi:10.1016/j.jhazmat.2013.06.032.

330. Wu Q, Su N, Cai J, Shen Z, Cui J. Hydrogen-rich water enhances cadmium tolerance in Chinese cabbage by reducing cadmium uptake and increasing antioxidant capacities. J Plant Physiol. 2015;175:174-82. doi:10.1016/ j.jplph.2014.09.017.

331. Cui W, Fang P, Zhu K, Mao Y, Gao C, Xie Y, et al. Hydrogen-rich water confers plant tolerance to mercury toxicity in alfalfa seedlings. Ecotoxicol Environ Saf. 2014;105:103-11. doi:10.1016/j.ecoenv.2014.04.009.

332. Sato Y, Kajiyama S, Amano A, Kondo Y, Sasaki T, Handa S, et al. Hydrogen-rich pure water prevents superoxide formation in brain slices of vitamin C-depleted SMP30/GNL knockout mice. Biochem Biophys Res Commun. 2008;375(3):346-50. doi:10.1016/j.bbrc.2008.08.020.

333. Ono H, Nishijima $Y$, Adachi $N$, Sakamoto $M$, Kudo $Y$, Kaneko $K$, et al. A basic study on molecular hydrogen $(\mathrm{H} 2)$ inhalation in acute cerebral ischemia patients for safety check with physiological parameters and measurement of blood H2 level. Med Gas Res. 2012;2(1):21. doi:10.1186/2045-9912-2-21.

334. Seo T, Kurokawa R, Sato B. A convenient method for determining the concentration of hydrogen in water: use of methylene blue with colloidal platinum. Med Gas Res. 2012;2:1. doi:10.1186/2045-9912-2-1.

335. Shimouchi A, Nose K, Shirai M, Kondo T. Estimation of molecular hydrogen consumption in the human whole body after the ingestion of hydrogen-rich water. Adv Exp Med Biol. 2012;737:245-50. doi:10.1007/978-1-4614-1566-4_36.

336. Shimouchi A, Nose K, Mizukami T, Che DC, Shirai M. Molecular hydrogen consumption in the human body during the inhalation of hydrogen gas. Adv Exp Med Biol. 2013;789:315-21. doi:10.1007/978-1-4614-7411-1_42

337. Liu C, Kurokawa R, Fujino M, Hirano S, Sato B, Li XK. Estimation of the hydrogen concentration in rat tissue using an airtight tube following the administration of hydrogen via various routes. Sci Rep. 2014;4:5485. doi:10.1038/srep05485.

338. Penders J, Kissner R, Koppenol WH. ONOOH does not react with $\mathrm{H} 2$ : Potential beneficial effects of $\mathrm{H} 2$ as an antioxidant by selective reaction with hydroxyl radicals and peroxynitrite. Free Radic Biol Med. 2014;75:191-4. doi:10.1016/j.freeradbiomed.2014.07.025.

339. Kato S, Matsuoka D, Miwa N. Antioxidant activities of nano-bubble hydrogen-dissolved water assessed by ESR and 2,2'-bipyridyl methods. Mater Sci Eng C Mater Biol Appl. 2015;53:7-10. doi:10.1016/ j.msec.2015.03.064

\section{Submit your next manuscript to BioMed Central and take full advantage of:}

- Convenient online submission

- Thorough peer review

- No space constraints or color figure charges

- Immediate publication on acceptance

- Inclusion in PubMed, CAS, Scopus and Google Scholar

- Research which is freely available for redistribution 